\title{
Sema3E-Plexin D1 signaling drives human cancer cell invasiveness and metastatic spreading in mice
}

\author{
Andrea Casazza, ${ }^{1}$ Veronica Finisguerra, ${ }^{2,3}$ Lorena Capparuccia, ${ }^{1}$ Andrea Camperi, ${ }^{1}$ \\ Jakub M. Swiercz, ${ }^{4}$ Sabrina Rizzolio, ${ }^{1}$ Charlotte Rolny, ${ }^{1}$ Claus Christensen, ${ }^{5}$ Andrea Bertotti, ${ }^{1}$ \\ Ivana Sarotto, ${ }^{1}$ Mauro Risio, ${ }^{1}$ Livio Trusolino, ${ }^{1}$ Jurgen Weitz, ${ }^{6}$ Martin Schneider, ${ }^{6}$ \\ Massimilano Mazzone, ${ }^{2,3}$ Paolo M. Comoglio, ${ }^{1}$ and Luca Tamagnone ${ }^{1}$
}

\begin{abstract}
${ }^{1}$ Institute for Cancer Research and Treatment (IRCC), University of Torino Medical School, Candiolo, Italy. ${ }^{2}$ Vesalius Research Center, Flanders Institute for Biotechnology (VIB), Leuven, Belgium. ${ }^{3}$ Vesalius Research Center, Katholieke Universiteit Leuven, Leuven, Belgium. ${ }^{4}$ Department of Pharmacology, Max-Planck Institute for Heart and Lung Research, Bad Nauheim, Germany. ${ }^{5}$ Danish Cancer Society, Institute of Cancer Biology, Department of Cancer Genetics, Copenhagen, Denmark. ${ }^{6}$ Department of General, Visceral and Transplantation Surgery, University of Heidelberg, Heidelberg, Germany.
\end{abstract}

\begin{abstract}
Semaphorin 3E (Sema3E) is a secreted molecule implicated in axonal path finding and inhibition of developmental and postischemic angiogenesis. Sema3E is also highly expressed in metastatic cancer cells, but its mechanistic role in tumor progression was not understood. Here we show that expression of Sema3E and its receptor Plexin D1 correlates with the metastatic progression of human tumors. Consistent with the clinical data, knocking down endogenous expression of either Sema3E or Plexin D1 in human metastatic carcinoma cells hampered their metastatic potential when injected into mice, while tumor growth was not markedly affected. Conversely, overexpression of exogenous Sema3E in cancer cells increased their invasiveness, transendothelial migration, and metastatic spreading, although it inhibited tumor vessel formation, resulting in reduced tumor growth in mice. The proinvasive and metastatic activity of Sema3E in tumor cells was dependent on transactivation of the Plexin D1-associated ErbB2/Neu oncogenic kinase. In sum, Sema3E-Plexin D1 signaling in cancer cells is crucially implicated in their metastatic behavior and may therefore be a promising target for strategies aimed at blocking tumor metastasis.
\end{abstract}

\section{Introduction}

Semaphorins are secreted and membrane-bound signals, originally implicated in the control of axonal wiring (1). They comprise a wide protein family and are involved in a range of functions, from tissue morphogenesis to the immune response, to cancer progression. High-affinity semaphorin receptors are found in the families of the plexins and of the neuropilins (2). The relevance of the small cytoplasmic domain of neuropilins in semaphorin signaling is unclear; however, the intracellular domain of plexins has been characterized for controlling R-Ras and RhoA activity and hence negatively regulates integrin-mediated adhesion and cell migration $(1,3)$. In addition, other molecules can interact with semaphorins or semaphorin receptors on the cell surface, featuring a complex scenario of multiple potential signaling pathways. In particular, tyrosine kinase receptors may become activated in response to semaphorins (4-7). This often leads to an apparent biological paradox whenever a semaphorin, in addition to mediating inhibitory signals (e.g., cell repulsion, inhibition of cell migration, etc.), can furthermore promote cell migration and invasive growth by activating tyrosine kinases in a cell-specific manner (8).

The role of semaphorin signalling in tumor progression is currently under close scrutiny. It is known that cancer cells release semaphorins regulating their own behavior as well as that of cells

Authorship note: Veronica Finisguerra, Lorena Capparuccia, and Andrea Camperi contributed equally to this work.

Conflict of interest: The authors have declared that no conflict of interests exists. Citation for this article: J Clin Invest. 2010;120(8):2684-2698. doi:10.1172/JCI42118. in the tumor microenvironment, such as endothelial cells and recruited leukocytes (see refs. 9 and 10 for reviews). For example, semaphorin 3B (Sema3B), Sema3F, and Sema4D have been shown to differently regulate tumor growth, tumor angiogenesis, and metastatic progression (11-16). Moreover, based on scattered evidence, semaphorin expression levels might discriminate among tumor cells with different metastatic abilities $(13,17,18)$. In this work, we focused on Sema3E (previously known as semaH), which was found to be overexpressed in metastatic tumor cells (17). We have previously demonstrated that, like other class 3 semaphorins, Sema3E is synthesized as a full-length precursor molecule of approximately $87 \mathrm{kDa}$ (p87-Sema3E), which is then subjected to proteolytic maturation by furin proprotein-convertases (PPCs), yielding a smaller fragment of approximately $61 \mathrm{kDa}$, referred to as $\mathrm{p} 61$. We have further shown that this proteolytic fragment promotes lung colonization of tumor cells injected in the circulation (19), but the implicated mechanisms remained unclear.

The high-affinity functional receptor of Sema3E is Plexin D1 and not any of the neuropilins (20). Genetic evidence showed that Sema3E-Plexin D1 signaling is required in developmental angiogenesis $(20,21)$. Moreover, recent evidence indicates that Sema3E is implicated in regulating postischemic angiogenesis (22). Intriguingly, while Plexin D1 expression is generally low in normal adult tissues, it is elevated in endothelial cells of tumor vessels and in cancer cells $(23,24)$. Like other family members, Plexin D1 is able to trigger R-Ras inactivation, leading to axonal and cell repulsion in vitro (25). Moreover, it was shown that Sema3E mediates either axonal attraction or repulsion in distinct neuronal populations, 


\section{Table 1}

Relative gene expression levels in human colorectal tumors and metastases

\begin{tabular}{lccl} 
Gene & \multicolumn{2}{c}{ Primary tumors } & Metastases \\
& M0 & M1 & \\
SEMA3E & $0.03 \pm 0.01$ & $0.42 \pm 0.19^{\mathrm{A}}$ & $0.65 \pm 0.17^{\mathrm{B}, \mathrm{C}}$ \\
PLXND1 & $7.16 \pm 1.89$ & $7.47 \pm 2.91$ & $20.4 \pm 3.15^{\mathrm{B}}$ \\
\hline
\end{tabular}

Quantitative RT-PCR data are presented as average \pm SEM of transcripts per $10^{4}$ copies $\beta$-actin ( $n=43 \mathrm{M} 0$ primary tumors, $n=17 \mathrm{M} 1$ primary tumors, and $n=51$ metastases) (see also Supplemental Figure $1, \mathrm{~A}-\mathrm{C})$. M0, nonmetastatic tumors; M1, tumors associated with metastatic disease. ${ }^{A} P<0.01$ versus $M 0$ primary tumors; ${ }^{B} P<0.0001$ versus primary tumors; ${ }^{C} P<0.01$ versus $\mathrm{M} 1$ primary tumors.

depending on the coexpression of Neuropilin-1 with Plexin D1 (26). This suggests that Sema3E can mediate divergent functions in different cells, depending on the implicated receptor complexes. Intriguingly, according to a recent report, Sema3E could inhibit the survival of MDA-MB-435 tumor cells in vitro but not their growth in vivo, while the opposite was true for MDA-MB-231 carcinoma cells (27); however, the mechanisms involved could not be elucidated. In the present work, we show that Sema3E is a double-faced signal in cancer development, eliciting 2 mirror effects in tumor cells and in the endothelial cells of tumor vessels, and further elucidate the implicated signaling pathways. Moreover, we show that Sema3E expression in human primary tumors is statistically associated with their metastatic progression and demonstrate in mouse preclinical models that interfering with Sema3E signaling in primary tumors results in a striking reduction of their metastatic dissemination.

\section{Results}

Sema3E expression correlates with the metastatic potential of human tumors. Sema3E was initially identified in mouse metastatic cancer cells (17). However, whether the expression of Sema3E or its receptor Plexin D1 in human primary tumors may be correlated with the metastatic progression has not been assessed. We analyzed gene expression in a wide series of 60 human colon carcinoma samples, for which we obtained detailed clinicopathological information (including the possible development of metastasis), as well as in a separate series of excised liver metastases (Supplemental Figure 1A; supplemental material available online with this article; doi:10.1172/JCI42118DS1). While the expression of the receptor Plexin D1 was clearly detected in all primary tumors, Sema3E levels were above detection threshold in only a fraction of them (independently from tumor grade or stages of local invasiveness; data not shown). Interestingly, Sema3E was expressed in $88 \%$ of the cases associated with metastatic disease (M1) and in only $46 \%$ of the nonmetastatic tumors (M0). This biased distribution was statistically significant, as confirmed by Fisher's exact test $(P=0.004)$. Consistently, as shown in Table 1, average Sema3E expression levels were significantly higher in metastatic versus nonmetastatic primary tumors $(P=0.002)$. Moreover, we could invariably detect Sema3E expression in $100 \%$ of liver metastases from colon carcinomas, and average levels of Sema3E and Plexin D1 were significantly higher in metastasis compared with primary tumors. Thus, Sema3E-Plexin D1 signaling seems to correlate positively with the metastatic progression of human colon carcinomas.
Interestingly, a recent immunohistochemistry analysis showed that Plexin D1 receptor levels are increased in invasive and metastatic melanomas, compared with low-grade tumors and benign nevi (28). Previous data on Sema3E expression in melanomas were controversial; thus, we analyzed a wide series of benign nevi, lowgrade melanomas, and highly invasive and metastatic melanomas, by using a polyclonal antibody previously validated for detecting all isoforms of Sema3E, including the proteolytically activated fragment p61 (19). We found that Sema3E was expressed in only a fraction of the melanocytes forming benign or dysplastic nevi as well as of the melanoma cells of low grade; in contrast, the percentage of Sema3E-positive cells increased to almost $100 \%$ in invasive and metastatic melanomas of Clark levels III and IV (Figure 1). Moreover, Sema3E expression at the single cell level seemed to be higher in malignant melanomas, compared with nevi. Thus, according to our data, also in melanoma progression, Sema3E signaling in primary tumors correlates with the potential for metastatic spreading.

Knocking down either Plexin D1 or Sema3E expression in cancer cells curtails metastatic spreading. We have demonstrated above that Sema3E levels directly correlate with the invasive and metastatic phenotype of human tumors of different histotype. We therefore investigated whether the genetic knockdown of either Sema3E (or its receptor Plexin D1) in validated experimental tumor models could affect the metastatic behavior. As shown in Figure 2A, we first silenced Plexin D1 expression in human metastatic carcinoma cells A549 (expressing endogenous Sema3E) and transplanted them in athymic mice to study tumor development and metastatic dissemination. Interestingly, Plexin D1-deficient tumors developed as controls (Figure 2B), suggesting that Plexin D1 expression in cancer cells do not have a major role in regulating growth or survival. In contrast, the number of lung metastases that spontaneously arose from Plexin D1-depleted primary tumors was significantly reduced (Figure $2 \mathrm{C}$ ), indicating a pivotal role of this receptor in the invasive-metastatic process.

In order to assess the functional relevance of endogenous Sema3E expression in tumor cells, we furthermore generated and transplanted in vivo Sema3E-deficient A549 cells (Figure 2, D-F). Analogously, we knocked down Sema3E expression in highly metastatic 4T1 breast carcinoma cells and carried out tumor transplantation experiments in syngenic mice, both subcutaneously and in the orthotopic site of mammary fat pad (Figure 2, G-L). Although the growth of the subcutaneous and orthotopic tumors was not significantly affected upon Sema3E silencing (Figure 2, H and J), their spontaneous metastatic dissemination was significantly reduced (Figure 2, I and K). Moreover, knocking down the endogenous Sema3E expression greatly reduced the metastatic potential of tumor cells injected in the systemic circulation (Figure 2L). These data were further validated using a second independent shRNA targeting the Sema3E transcript (Supplemental Figure 2, A and B). In addition, and consistent with our expression data in human tumor samples, we found that the metastatic potential of HCT-116 colon carcinoma cells was significantly reduced upon Sema3E knockdown (Supplemental Figure 2C). Altogether, our results indicate that the expression of both Sema3E and Plexin D1 is crucially involved in the metastatic spreading of diverse cancer cells.

Sema3E overexpression inhibits tumor vessel density and tumor growth but promotes metastatic spreading. Prompted by the results reported above, we questioned whether a gain of Sema3E expression could in turn enhance the metastatic potential of tumor cells. Three human cancer cell lines (i.e., HCT-116 colon carcinoma, MDA-MB-435 

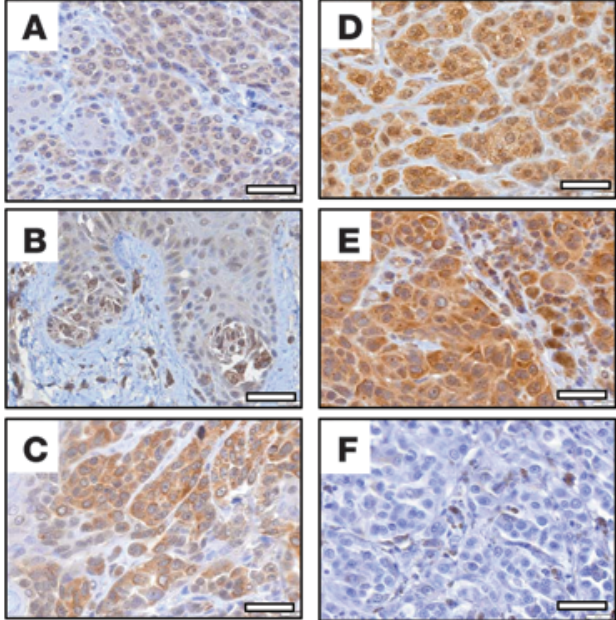

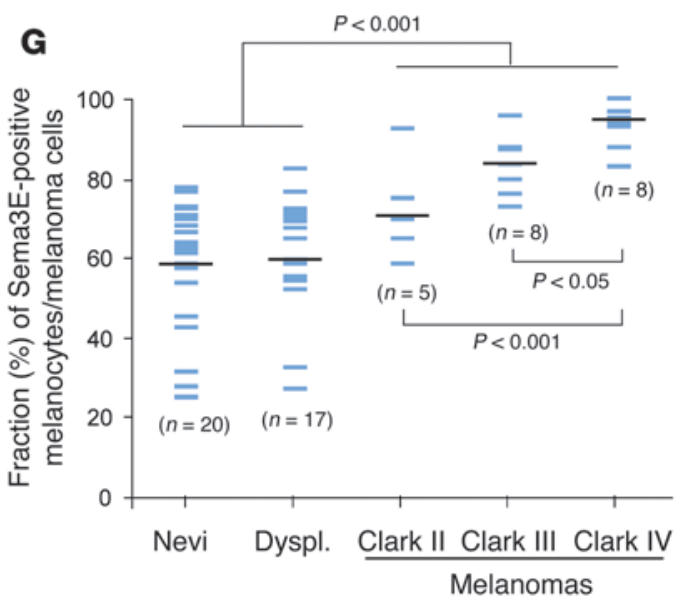

\section{Figure 1}

Elevated Sema3E expression in invasive human melanomas. (A-F) Sema3E expression was detected by immunohistochemistry in a series of 58 nevi and melanoma human samples, representing different stages of progression. Micrographs show representative fields of Sema3Eexpressing samples: (A) an intradermal nevus, (B) a dysplastic nevus, (C) a Clark III level malignant melanoma, and (D and E) 2 different Clark IV level melanomas. (F) Control staining of a Clark IV melanoma, without including the specific antibody. Scale bar: $40 \mu \mathrm{m}$. (G) In the graph, each case is represented by a blue bar, indicating the fraction of Sema3E-positive cells in the sample (scored as described in Methods). Black bars indicate the mean value for each of 5 groups (intradermal-junctional nevi [nevi], dysplastic nevi/melanomas in situ [dyspl.], and Clark II, Clark III, and Clark IV level melanomas).

melanoma, and A549 lung carcinoma) were therefore transduced with lentiviral vectors encoding Sema3E or empty vector $(\mathrm{EV})$ controls. Overexpressing cells contained a small amount of the fulllength Sema3E precursor of approximately $87 \mathrm{kDa}$ (p87-Sema3E) as well as a predominant amount of the matured fragment p61 (Supplemental Figure 3A and data not shown), indicating an efficient spontaneous proteolytic conversion. Notably, p61 was also the main species of endogenous Sema3E released by cancer cells (including those used for our gene knockdown experiments; see Supplemental Figure 3B). Moreover, we found that the p61 fragment was fully competent to bind the receptor Plexin D1 and elicit the typical collapsing response (Supplemental Figure 3, C and D). For these reasons, in many subsequent experiments, a recombinant form of the matured p61 fragment was also used.

Sema3E overexpression did not elicit morphological changes in cancer cells (data not shown), and we did not observe any significant difference in viability and proliferation in culture (Supplemental Figure 4A). Sema3E-expressing and control cells were then transplanted subcutaneously in nude mice to establish primary tumors. Remarkably, the elevated expression of Sema3E led to a significant reduction in tumor volume and weight in both models (Figure 3A and Supplemental Figure 4B). By assessing the mitotic index (with Ki-67; Figure 3B) in tumor tissue sections, we found that Sema3E expression did not significantly affect tumor cell proliferation, consistent with in vitro analysis. However, cellular apoptosis in Sema3E-expressing tumors was increased by approximately 2 fold, as revealed by TUNEL (Figure 3C) and further confirmed by the detection of the apoptotic markers cleaved caspase-9 and lamin B (Figure 3D). Notably, we found that the vascular density of Sema3E-expressing tumors was significantly reduced (Figure 3E); moreover, Sema3E had significantly induced endothelial cell apoptosis (Figure 3F). These data suggested a potential paracrine activity in the microenvironment of the semaphorin released by tumor cells.
When looking at the metastatic spreading from these primary tumors, we found that - despite their reduced size - tumors overexpressing Sema3E gave rise to a strikingly increased number of spontaneous lung metastases (Figure 4A) expressing Sema3E (Supplemental Figure 5A), which indicated a predominant effect of the semaphorin to enhance the invasive-metastatic process. Notably, tumor xenografts overexpressing full-length processable Sema3E mainly contained mature p61 fragments (Supplemental Figure 5B), further confirming in vivo an efficient proteolytic conversion of the precursor. Moreover, the expression of a recombinant p61 fragment in tumors resulted in comparable effects in vivo, as observed with wild-type processable Sema3E undergoing spontaneous proteolytic conversion (Supplemental Figure 5, C-E, and Figure 4B).

To establish the functional relevance in vivo of Sema3E signaling in cancer cells, as opposed to its potential paracrine activity on endothelial cells, we knocked down the expression of the receptor Plexin D1 in MDA-MB-435 cells. Plexin D1-silenced cancer cells were viable and did not display any morphological change in culture (data not shown). Importantly, we found that Plexin D1 knockdown in cancer cells did not prevent the inhibitory effect of p61-Sema3E on tumor growth (Figure 4C), tumor vessel density (Figure 4D), or endothelial cell apoptosis (data not shown), supporting the idea that secreted Sema3E acts on the tumor endothelium in a paracrine manner. In contrast, p61-Sema3E overexpression could not induce the metastatic behavior of these Plexin D1-deficient cells (Figure 4E). Thus, the prometastatic activity of Sema3E is a tumor cell-autonomous, Plexin D1-dependent effect, independent from the paracrine activity capable of endothelial cells repulsion. These results could explain the apparent paradox observed in our experimental tumors in vivo and suggested 2 independent functions mediated by p61Sema3E in different cell types, via the same receptor, Plexin D1.

Overall, forced overexpression of Sema3E in cancer cells upholds a paracrine antiangiogenic effect and leads to reduced tumor growth, a functional effect which was not observed with endog- 
A

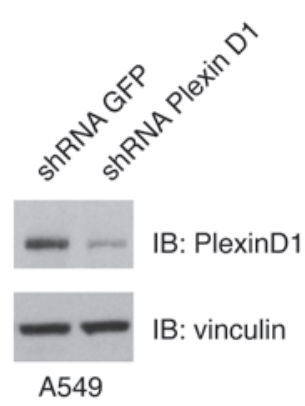

D

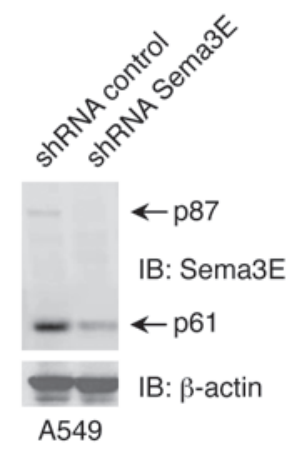

G

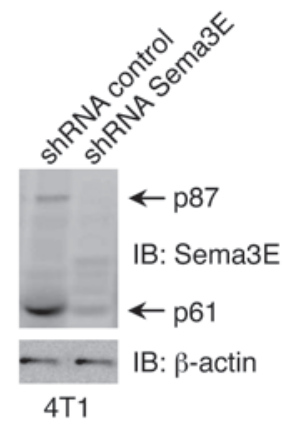

J

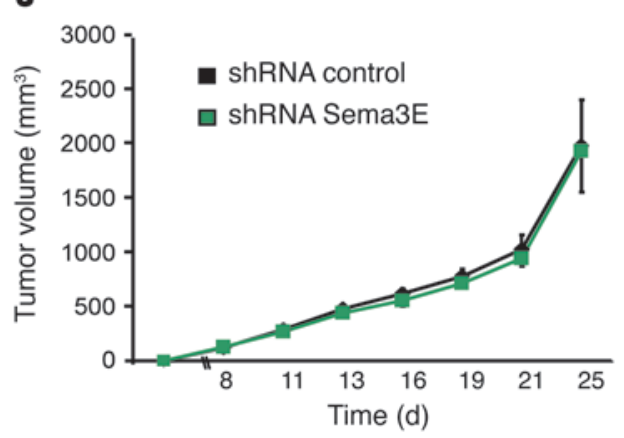

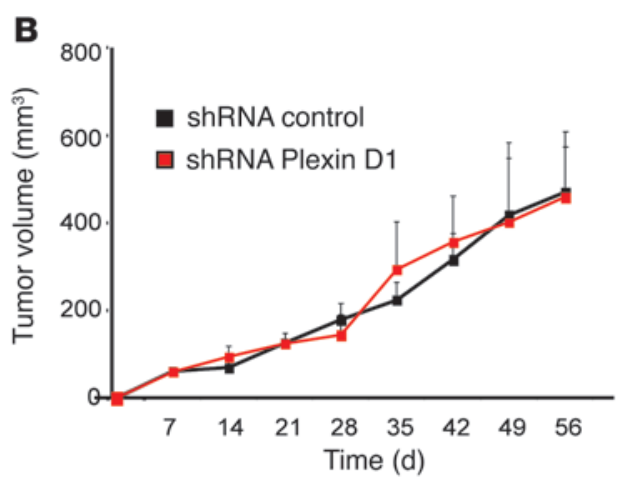
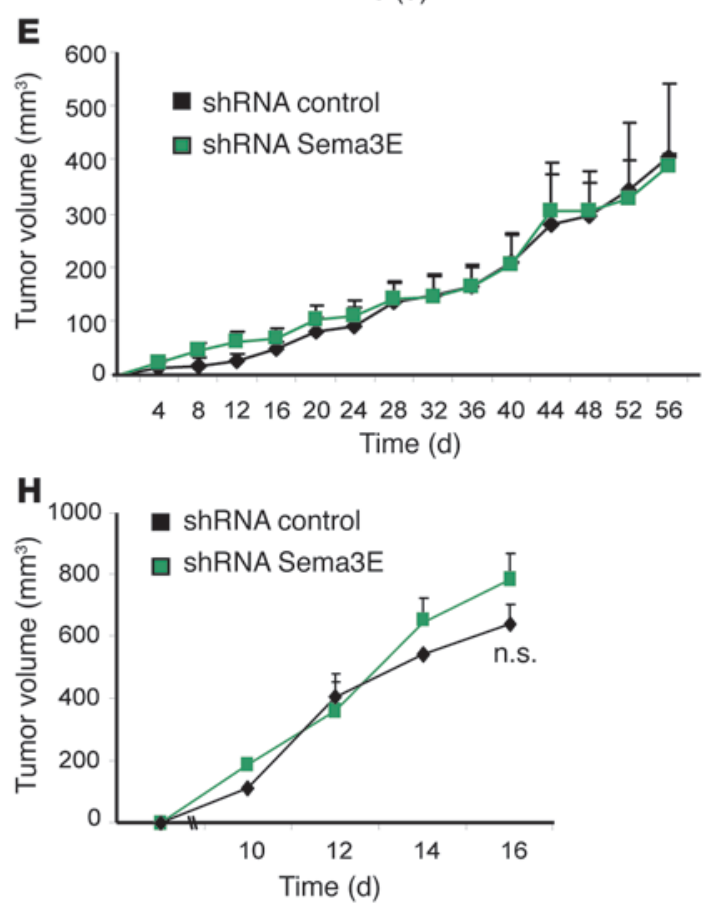

K

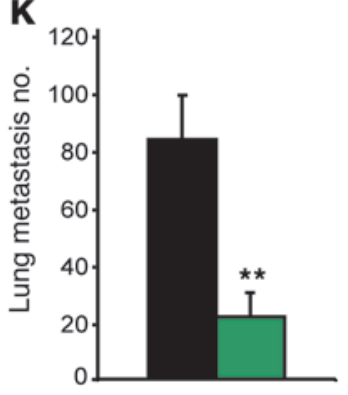

C

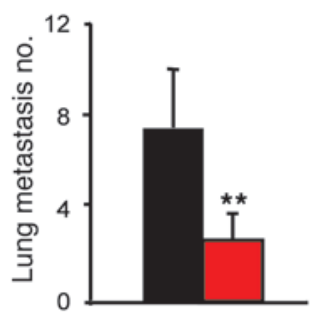

$\mathbf{F}$

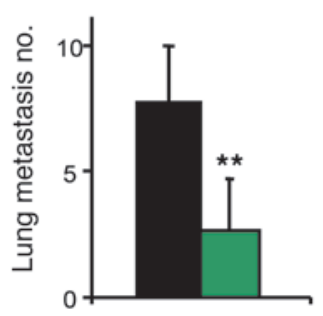

I

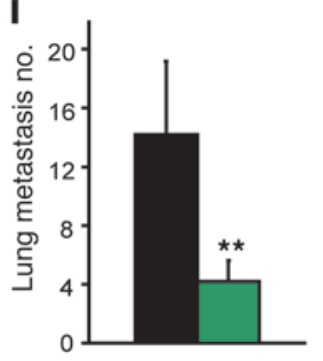

L

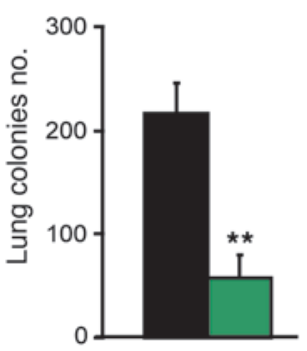

Figure 2

Plexin D1 and Sema3E knockdown in tumor cells inhibits the metastatic spreading. (A-C) A549 carcinoma cells were transduced to stably express shRNAs targeting Plexin D1 (or unrelated control sequences), and the expression knockdown was verified by Q-PCR (data not shown) and (A) Western blotting. Plexin D1-deficient and control cells were then transplanted into nude mice to establish subcutaneous tumors. Throughout the graphs, (B, E, H, and $\mathbf{J})$ display the growth of primary tumors and $(\mathbf{C}, \mathbf{F}, \mathbf{I}$, and $\mathbf{K})$ the number of spontaneous lung metastases scored at the end of the experiment. Data are given as average \pm SD of 6 mice for each experimental group throughout. (C) ${ }^{* \star} P<0.005$. (D-F) A549 cells were transduced to stably express shRNAs targeting Sema3E (or unrelated control sequences), and gene knockdown was verified by Q-PCR (data not shown) and (D) Western blotting. ${ }^{* \star} P<0.003$. The expression of a second independent shRNA targeting Sema3E yielded similar results (see Supplemental Figure 2, A and B). (G-I) Analogous experiment as above, using Sema3E-depleted 4T1 cells injected subcutaneously into syngenic Balb/c mice. ${ }^{* *} P<0.007$. ( $\mathbf{J}$ and $\mathbf{K}$ ) The same Sema3E-depleted $4 \mathrm{~T} 1$ cells as above were injected orthotopically in the fat pad of Balb/c mice. Data are given as the average \pm SD of 6 mice for each experimental group. ${ }^{\star \star} P<0.003$. (L) The same Sema3E-deficient 4T1 cells (as in G-K) were directly injected intravenously in Balb/c mice, and the number of lung metastatic colonies was evaluated as above. ${ }^{\star \star} P<0.004$. 
enous expression levels. On the other hand, both gene knockdown and overexpression experiments indicated that the Sema3E-dependent prometastatic effect depends on a distinctive signalling cascade into tumor cells, mediated by the Plexin D1 receptor.

Sema3E-dependent endothelial cell repulsion relies on Rnd2-expression and $R$-Ras inactivation. In an attempt to unravel the mechanisms underlying the effects of Sema3E on tumor vessels and metastasis, we performed in vitro experiments analyzing endothelial and tumor cells, respectively.

Functional assays in culture demonstrated that Sema3E (and its matured fragment p61) was capable of inhibiting the migration of HUVECs in a Plexin D1-dependent manner and eliciting their repulsion and cellular collapse (Figure 5, A and B, and Supplemental Figure 6, A and B). Moreover, in a mixed-culture experiment reminiscent of the in vivo setting, we found that Sema3E-expressing tumor cells could efficiently induce endothelial cell repulsion only if the latter contained Plexin D1 (Supplemental Figure 6B).

It was recently shown that cell collapse and axonal repulsion mediated by Plexin D1 depend on its intrinsic R-Ras GAP activity (25). To investigate the relevance of this pathway in endothelial cells treated with Sema3E, we overexpressed a dominant-active mutant of R-Ras that cannot be regulated by Plexin D1 (25) and found that endothelial cells were no longer sensitive to p61dependent inhibition of migration (Figure 5C). Moreover, it was reported that the GAP activity of Plexin D1 depends on the association of the small GTPase Rnd2 (25). Notably, we found that Rnd2 is abundantly expressed in HUVECs (Supplemental Figure 6C); moreover, upon knocking down Rnd2 expression, these cells became insensitive to Sema3E-mediated inhibition of migration (Figure 5D) and cellular collapse (Figure 5E). These data indicate that the inhibitory function of Sema3E in endothelial cells is dependent on the R-Ras GAP activity of Plexin D1 and on their prominent expression of Rnd2. Altogether, these data are consistent with the reported restrictive role of Sema3E in developmental and postischemic angiogenesis $(20,22)$ and with our in vivo data indicating inhibition of tumor vasculature.

p61-Sema3E signaling in tumor cells promotes motility and invasiveness, transendothelial migration, and extravasation in vivo. We aimed at elucidating the molecular mechanisms responsible for the prometastatic activity of p61-Sema3E. As demonstrated above in Figure 4E, our experimental evidence indicates that this function crucially depends on signaling cascades deployed into the tumor cells, which could potentially regulate their intrinsic migratory and invasive/ metastatic behavior. In keeping with this hypothesis, we found that different cancer cells were induced to migrate in response to p61Sema3E, either provided in the environment (Figure 6A) or constitutively overexpressed in autocrine manner (Figure 6B); moreover, this function was dependent on endogenous Plexin D1 receptor expression (Figure 6B). Notably, unlike HUVECs, none of the cancer cells analyzed underwent the collapsing response upon Sema3E treatment (Supplemental Figure 6A and data not shown), possibly reflecting their relatively low expression of the required mediator Rnd2 (see Supplemental Figure 6C). Moreover, knocking down Rnd 2 expression did not impair Sema3E-induced cancer cell migration (Supplemental Figure 10A). Thus, cancer cells and endothelial cells respond differently to Sema3E/Plexin D1 signaling.

Further elucidating the mechanisms involved in the prometastatic activity of p61-Sema3E in tumor cells, we found that it promotes the invasion of the ECM (Figure 6C). Moreover, p61 expression induced ECM degradation spots, colocalizing with actin-enriched focal structures (Supplemental Figure 7), resembling adhesive contacts called invadopodia, which are known to mediate the localized release of ECM-degrading enzymes (29). Notably, ECM degradation and invadopodia are instrumental for cancer cells slithering in and out blood vessel walls in order to metastasize. To address the potential involvement of p61-Sema3E in tumor cell extravasation, we initially exploited a transendothelial migration assay in vitro. We found that p61-expressing tumor cells, unlike controls, promptly migrated through a compact monolayer of endothelial cells (Figure 6D). Importantly, p61induced transendothelial migration was abrogated in cancer cells lacking Plexin D1 receptor (Figure 6D).

It is known that a limited fraction of the cells released in the circulation by primary tumors forms metastases. Some of these cells die soon after entering the blood stream; others get entrapped in narrow capillary beds (e.g., in the lungs) and die in thrombotic emboli or manage to return in the circulation. A minority of circulating cancer cells will manage to extravasate, eventually forming metastatic colonies. In order to elucidate in vivo the role of Sema3E in tumor cell extravasation, we injected intravenously fluorescence-labeled MDA-MB-435 cells expressing p61 (or EV-controls) and subsequently sacrificed mice at different time points (from 15 minutes to 48 hours after injection) to quantify metastatic spreading. Initially, as expected, tumor cells accumulated in the lungs, independent from their expression of p61-Sema3E (Figure 7A) and consistent with the mechanical entrapment in the first encountered capillary bed. As expected, the number of cancer cells present in the lungs gradually decreased over time. However, by 1 hour after injection, p61Sema3E-expressing cells were found in strikingly greater number compared with controls. At 48 hours after injection, 10-fold more p61-expressing cells were present in the lungs. This is consistent with the fact that p61- expressing cells directly injected in the circulation gave origin to a larger number of metastatic lung colonies, as scored in long term experiments (Supplemental Figure 8A). Moreover, similar experiments were performed by using Plexin D1-silenced cancer cells. In this case, p61Sema3E could not induce the metastatic behavior (Figure 7B), thereby confirming that the proinvasive and prometastatic activity displayed by p61-Sema3E is dependent on a signaling cascade intrinsic to tumor cells.

Furthermore, we used a drug-inducible system to control semaphorin expression in tumor cells injected in vivo (see Supplemental Methods for details). Two groups of mice received cells in which p61-Sema3E expression had been induced before injection; for one group drug treatment was maintained until the end of the experiment, whereas for the other group, treatment was stopped 4 days after injection (in order to terminate p61 expression after extravasation in lungs had occurred). Other mice received untreated cells, and p61 expression began only 4 days after injection. One month later, the lungs were analyzed to score the presence of metastases. We found that p61-Sema3E expression in cancer cells, at the time of injection, resulted in a 10 -fold increase in the number of metastatic colonies compared with that of controls (even if druginduced expression was terminated soon after). On the contrary, the induction of semaphorin expression after the extravasation step only produced a negligible increase in the number of detectable lung colonies compared with that of controls (Supplemental Figure 8B). These data strongly suggested that p61-Sema3E mainly acts by promoting tumor cell extravasation. 
A

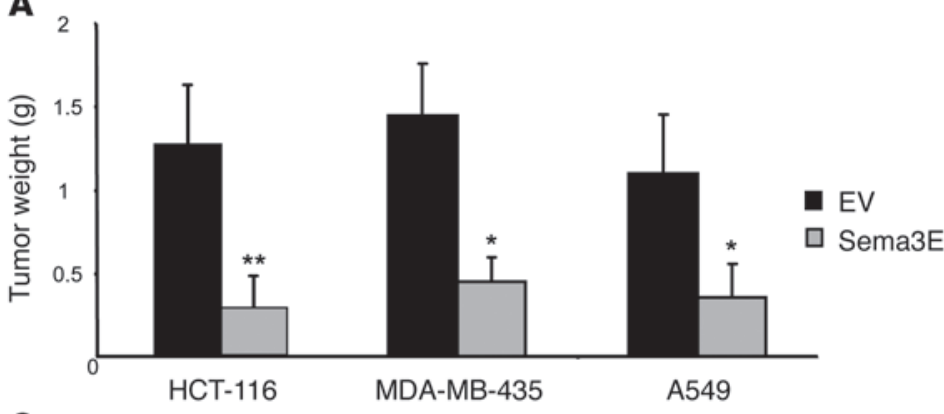

B

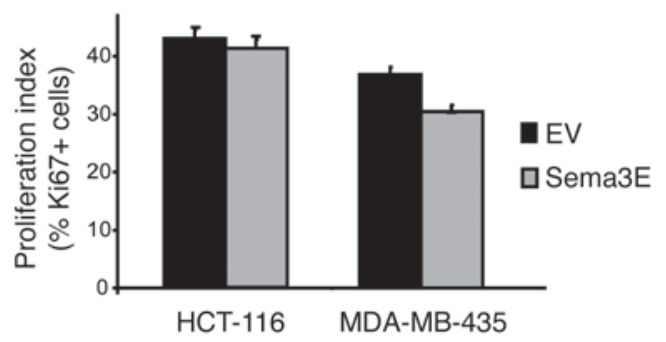

C

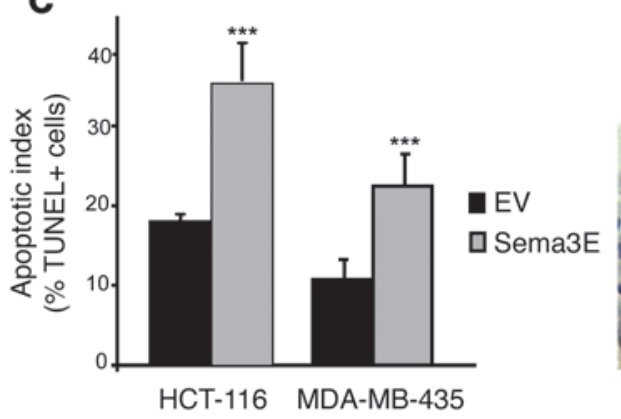

E

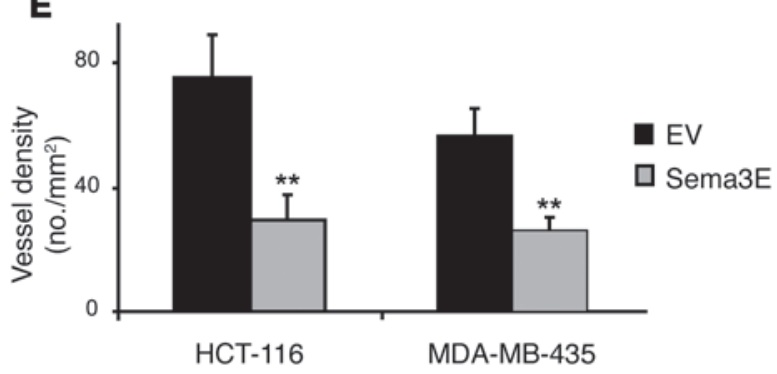

EV

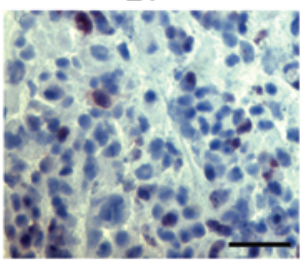

Sema3E

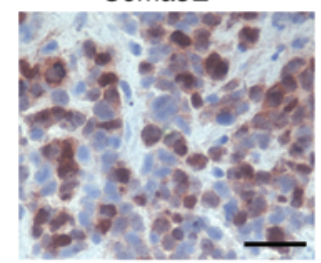

D

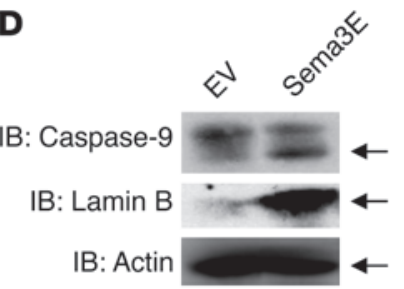

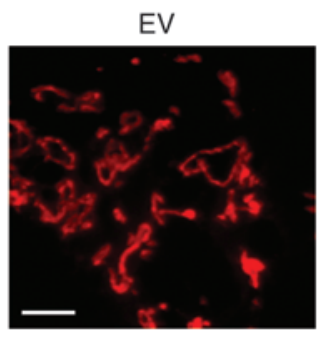

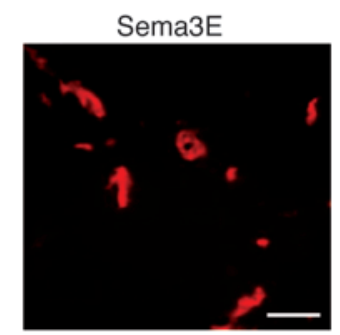

$F$

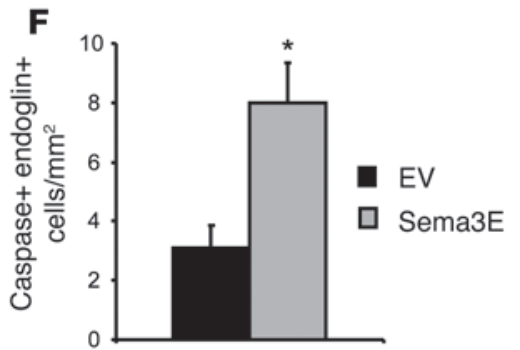

Figure 3

Sema3E overexpression inhibits tumor vessel density and tumor growth. (A) Sema3E-overexpressing and EV-control cancer cells of different histotypes were transplanted subcutaneously in nude mice. The growth of primary tumors was followed by external measuring (shown in Supplemental Figure 4B). Values in the graph indicate tumor weight at the end of the experiment. Data shown represent the average \pm SD of 6 mice per each experimental condition. ${ }^{*} P<0.01$; ${ }^{* \star} P<0.005$. (B) Control (EV) and Sema3E-expressing tumor sections (as indicated) were immunostained to detect Ki-67 proliferation marker. The graph indicates the percentage of KI-67-positive cells (average \pm SD). (C) Tumor sections (as above) were analyzed to reveal apoptotic cells by TUNEL. Data in the graph show the percentage of TUNEL-positive cells (average \pm SD). ${ }_{\star \star \star *} P<0.001$. Two representative fields of HCT-116 tumors are shown. Nuclei were visualized by hematoxylin. Scale bar: $30 \mu \mathrm{m}$. (D) Western blot analyses, revealing 2 different apoptotic markers in lysates of Sema3E-expressing tumors, i.e., cleaved caspase- 9 and the presence of lamin B in the cytosolic fraction. (E) Tumor sections were stained with anti-CD31 to reveal endothelial cells (in red). Values in the graph indicate the vessel density (average \pm SD). ${ }^{* \star} P<0.005$. Scale bar: $40 \mu \mathrm{m}$. (F) Apoptotic endothelial cells in tumors were identified by double staining with endoglin (red) and activated caspase-3 (green) markers. ${ }^{*} P<0.02$. Scale bar: $10 \mu \mathrm{m}$.

The prometastatic activity of $p 61-S e m a 3 E$ is mediated by the transactivation of Plexin D1-associated ErbB2. Other semaphorins, including Sema4D and Sema6D, have been shown to elicit cancer cell migration and invasive growth (4-6). Interestingly, the implicated receptors (i.e., Plexin B1 and Plexin A1) interact with receptor tyrosine kinases (RTKs) and trigger their distinctive signal pathways and functional outcomes. By analogy, p61-Sema3E could elicit the activation of a RTK associated with Plexin D1, putatively responsible for the observed prometastatic effect. We therefore undertook an unbiased screening approach to search for potential can- 
A
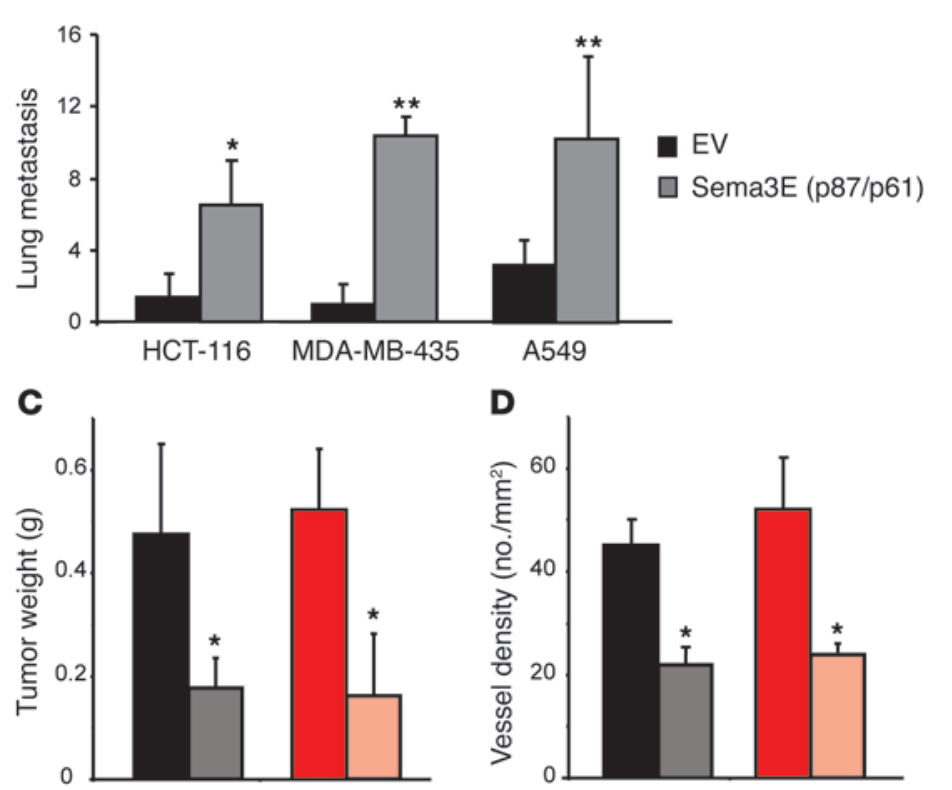

Plxnd1 mRNA fold changes
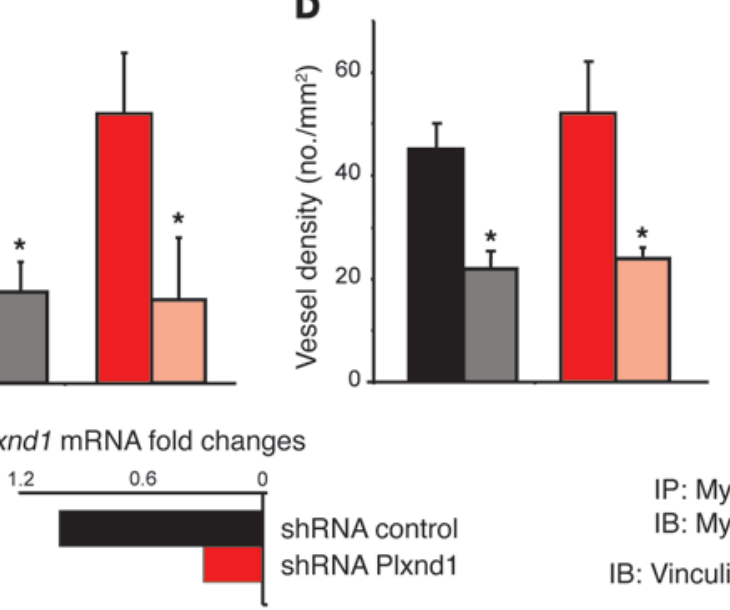

B

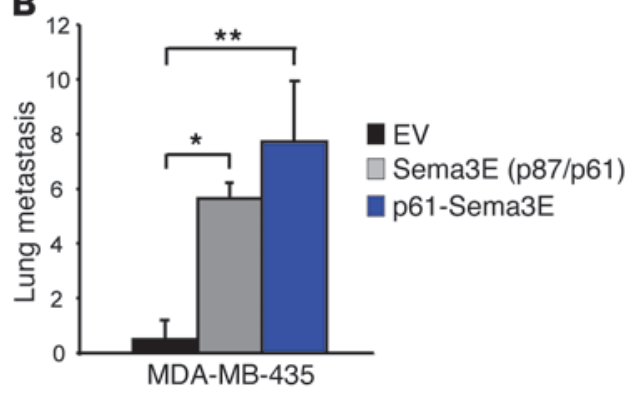

$\mathbf{E}$

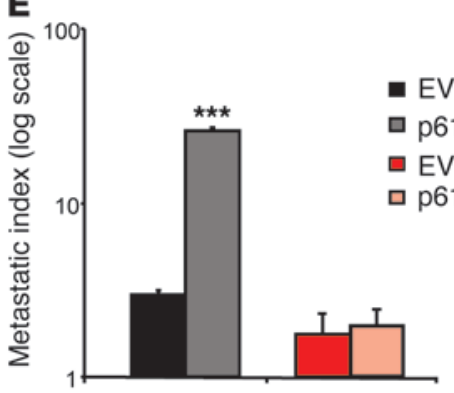

- EV $\mid$ shRNA control

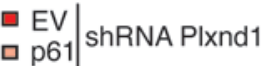

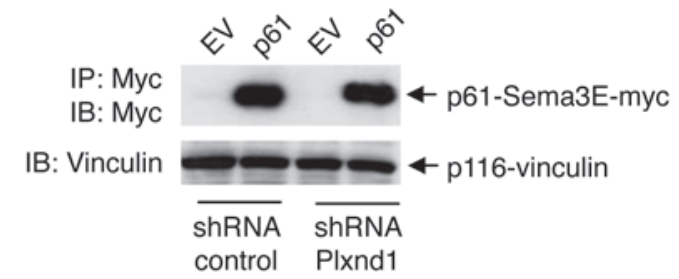

control Plxnd1

\section{Figure 4}

Sema3E-p61 signaling in cancer cells enhances the metastatic spreading. (A) Superficial metastases in the lungs of mice bearing subcutaneous tumor xenografts formed by control or Sema3E-expressing HCT-116, MDA-MB-435, or A549 cells. Data on primary tumors are displayed in Figure 3 and Supplemental Figure 4B. The graph indicates average values \pm SD of 6 mice per each experimental condition throughout the figure. ${ }^{\star} P<0.04 ;{ }^{* *} P<0.005$. (B) As above, we scored lung metastases in mice transplanted with MDA-MB-435 cancer cells expressing either processable Sema3E (largely converted into p61 in vivo) or the truncated recombinant p61 isoform. Data on primary tumors are shown in Supplemental Figure 5, C-E. ${ }^{*} P<0.02$; ${ }^{* *} P<0.005$. (C-E) Plexin D1-deficient MDA-MB-435 cancer cells (or controls), either expressing p61-Sema3E or EV (expression analysis is shown on the bottom) were injected subcutaneously in nude mice to form tumor xenografts. (C) Primary tumors carrying p61-Sema3E grew significantly less compared with controls and (D) displayed a reduced vessel density, independently from the expression of Plexin D1 in cancer cells, thereby suggesting a paracrine activity of the semaphorin in the microenvironment. (E) Conversely, p61 expression could not induce the metastatic spreading of tumor cells devoid of Plexin D1. ${ }^{*} P<0.05$; ${ }^{* \star} P<0.0005$.

didates in a low-density array, designed to simultaneously detect the tyrosine phosphorylation of 42 different RTKs in cell lysates. Interestingly, we found that ErbB2 phosphorylation was selectively increased in A549 carcinoma cells in response to p61-Sema3E (Supplemental Figure 9). ErbB2 is an oncogenic receptor often implicated in cancer invasion and metastasis; it has no cognate ligand but commonly becomes activated by forming heterodimers with other receptors of the same family (such as EGFR or ErbB3) (30-32). We have therefore individually analyzed immunopurified ErbB2 (Figure 8A) and the homologous ErbB3 and EGFR tyrosine kinases (Figure $8 \mathrm{~B}$ ) in cells treated with p61-Sema3E. In addition to that of ErbB2, we observed a minor increase in EGFR tyrosine phosphorylation induced by p61, while ErbB3 was not affected. ErbB2 phosphorylation in response to $\mathrm{p} 61$ was further confirmed in HeLa (Figure 8C) and MDA-MB-435 cells (data not shown). Notably, while p61 cannot bind ErbB2 (data not shown), it elicited ErbB2 phosphorylation in a Plexin D1-dependent manner, as demonstrated by receptor knockdown (Figure 8C).
Interestingly, we could copurify Plexin D1 and ErbB2 from lysates of overexpressing cells (Figure 8D), suggesting that they can form receptor complexes on the cell surface. Moreover, Plexin D1 became tyrosine phosphorylated in this complex upon p61 stimulation (Figure 8E), and this was impaired by an ErbB2 kinase inhibitor but not upon inhibition of the unrelated RTK Met (Figure 8E). Finally, and most importantly, we found that the 2 endogenous receptors Plexin D1 and ErbB2 associated in a complex in tumor cells, upon stimulation with p61-Sema3E (Figure 8F).

We then found that p61-induced migration of cancer cells was impaired upon inhibition of ErbB2 catalytic activity (Figure 9A and Supplemental Figure 11, A and B). Moreover, p61-expressing cells were unable to give rise to lung metastatic colonies upon systemic treatment of recipient mice with ErbB2 inhibitor (Figure 9B). Furthermore, by knocking down ErbB2 expression in cancer cells with RNAi technology, we could selectively impair p61-Sema3E signaling, both in vitro and in vivo. In fact, while ErbB2-depleted cells were viable and sensitive to motogenic signals, like hepa- 

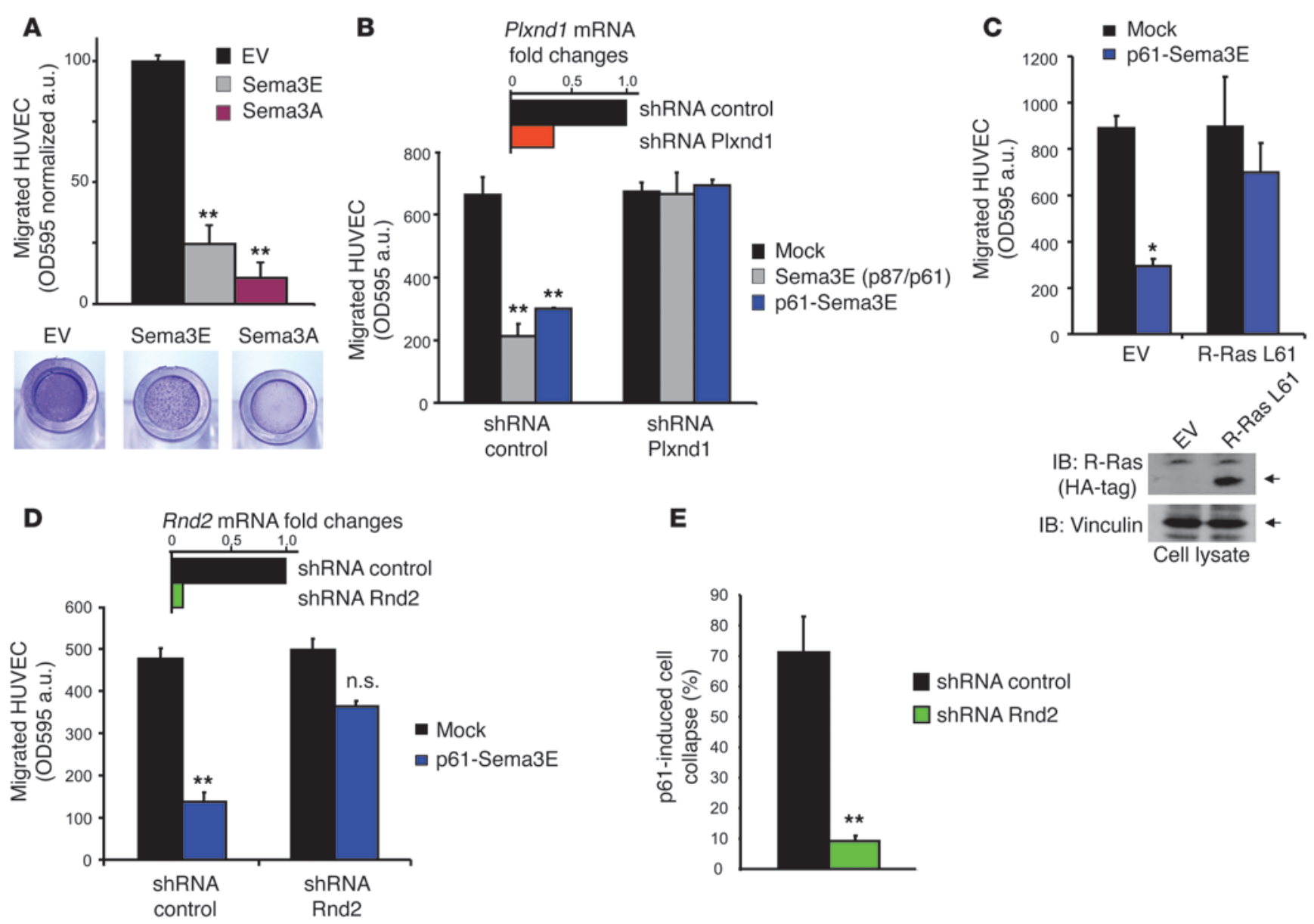

E

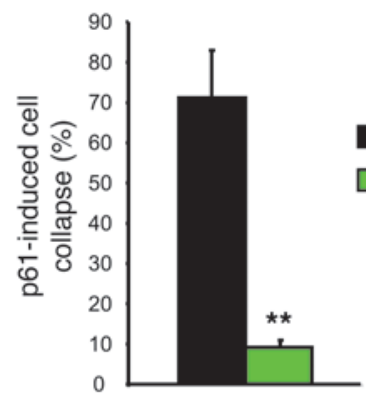

shRNA control

shRNA Rnd2

Figure 5

Molecular mechanisms of Sema3E-dependent endothelial cell repulsion. (A) The haptotactic migration of HUVECs was analyzed in the presence of $7 \mathrm{nM}$ purified Sema3E (or equal concentration of Sema3A, as positive control) in the lower chamber. Migrated cells were stained with crystal violet and quantified by absorbance at $595 \mathrm{~nm}$ (see Methods for details). The graphs show the average \pm SD of at least 2 independent experiments throughout the figure. ${ }^{\star \star} P<0.005$. See also Supplemental Figure 6, A and B. (B) Plexin D1 expression was knocked down in HUVECs (verified by Q-PCR), and the haptotactic migration of these cells was assayed in Transwell inserts. Both processable wild-type Sema3E (yielding a p87/p61 mix) and the recombinant p61-Sema3E fragment (7 nM each) inhibited the migration of controls but not of Plexin D1-deficient cells. Migrated cells were quantified by staining with crystal violet and measuring absorbance at $595 \mathrm{~nm}$ (see Methods). Data are given as average \pm SD of 2 different experiments. ${ }^{* *} P<0.005$. (C) HUVEC migration assay as above, upon expression of dominant-activated R-Ras-L61 mutant (verified by Western blotting). ${ }^{*} P<0.02$. (D) Rnd2 expression was knocked down in HUVECs by siRNA transfection (verified by Q-PCR), and cell migration was assayed as above. ${ }^{*} P<0.005$. (E) Rnd2-depleted HUVECs (same as above) were incubated with $7 \mathrm{nM}$ p61-Sema3E for 4 hours, then fixed and stained with FITC-Phalloidin. The percentage of collapsed cells (defined as having a diameter shorter than $30 \mu \mathrm{m}$ ) is indicated on the graph. Data are representative of at least 3 experiments, displaying consistent results. ${ }^{* *} P<0.005$.

tocyte growth factor, they did not respond to $\mathrm{p} 61$ in migration assays (Figure 9C) or in experimental metastasis assays in vivo (Figure 9D). Taken together, these data indicated that ErbB2 plays a master role in the invasive-metastatic properties orchestrated by p61-Sema3E and its receptor.

ErbB2-mediated signaling is well characterized, and MAPK, PI3KAKT, and PLC- $\gamma$ appear to be the main players $(30,31)$. Interestingly, MAPK and PLC- $\gamma$ cascades are typically activated in response to EGFR-ErbB2 heterodimers, while the PI3K-AKT pathway is the main mediator of ErbB2-ErbB3 complexes. We therefore analyzed the individual contribution of these signal transducers in Sema3Einduced tumor cell migration by applying the selective inhibitors LY294002, PD98059, and U-73122. As shown in Figure 9E, MAPK and PLC- $\gamma$ activation appeared to be required for function, while the PI3K-AKT pathway was only marginally implicated. Moreover, our analysis of MAPK, AKT, and PLC- $\gamma$ phosphorylation in tumor cells treated with Sema3E indicated a major activation of MAPK and PLC- $\gamma$ (Supplemental Figure 12). Thus, our data suggest that Sema3E/Plexin D1-dependent activation of a signaling cascade downstream to ErbB2 phosphorylation, especially via MAPK and PLC- $\gamma$ activation, is mainly responsible for the observed proinvasive and prometastatic function.

\section{Discussion}

Semaphorins and their receptors have been implicated in a range of functions, including angiogenesis and tumor progression $(9,10)$. In particular, the functional relevance of Sema3E in cancer was poorly understood, and the implicated signaling mechanisms had 


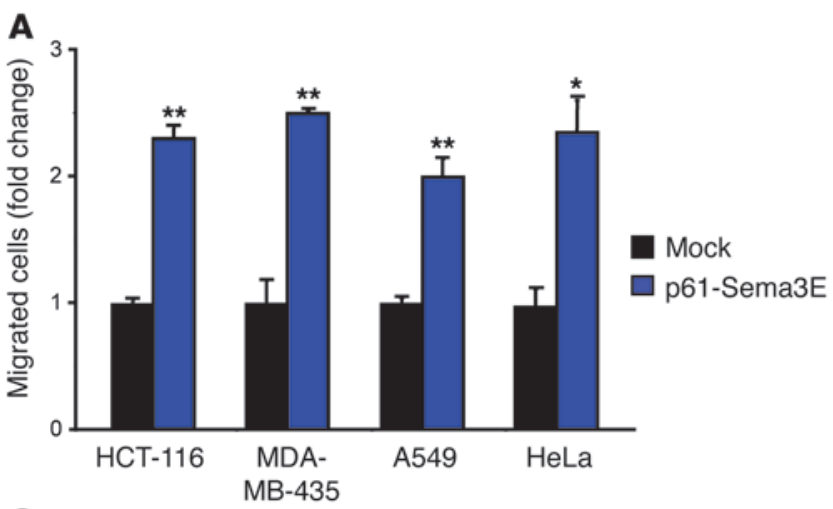

C

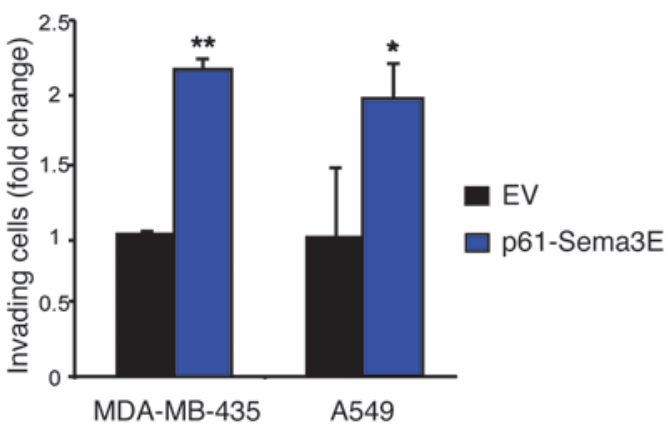

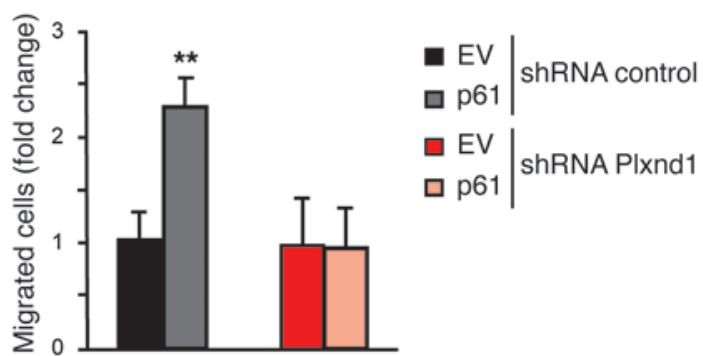

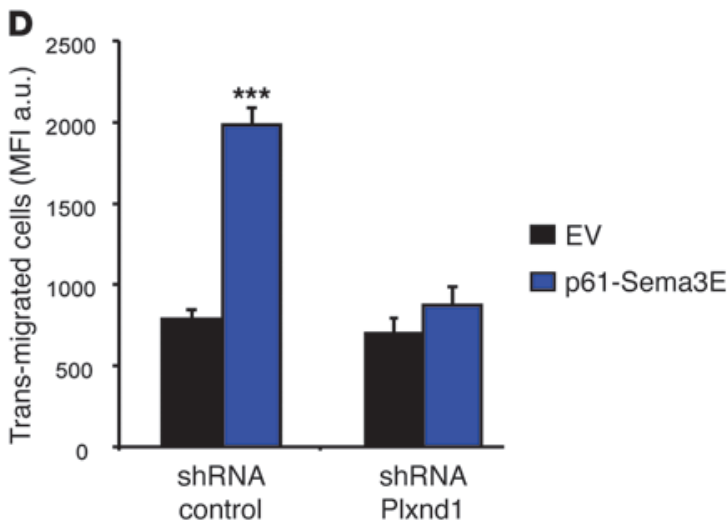

Figure 6

p61-Sema3E induces tumor cell migration and invasiveness. (A) The migration of the 4 indicated tumor cell lines was assayed in Transwell inserts in response to purified $7 \mathrm{nM}$ p61-Sema3E (included in the lower chamber). Migrated cells were quantified by staining with crystal violet (see Methods). Data are given as average $\pm \mathrm{SD}$ of at least 2 different experiments and values were normalized to respective controls. ${ }^{*} P<0.03$; ${ }^{\star *} P<0.005$. (B) MDA-MB-435 cells transduced with p61-Sema3E or EV and subjected to Plexin D1 knockdown by RNAi (the same as in Figure 4, $\mathrm{C}-\mathrm{E})$. Cell migration was assessed as above, and data were normalized to EV-shRNA control. ${ }^{* *} P<0.005$. Analogous results were obtained with HCT-116 cells (data not shown). (C) MDA-MB-435 or A549 tumor cells were added in the upper chamber of a Transwell insert coated with Matrigel and allowed to invade the matrix and migrate to the lower side of the porous membrane (quantified as above). Data shown are the average \pm SD of 2 different experiments. ${ }^{*} P<0.05$; ${ }^{* *} P<0.005$. (D) We assessed the transmigration of p61-expressing (or control) MDA-MB-435 cells (as in B) through a confluent monolayer of HUVECs (see Methods for experimental details). Tumor cells were labeled with CFDA-SE before the experiment. Transmigrated cells were detected by a fluorescence microscope and quantified using METAMORPH software. The graph shows the MFI \pm SD in triplicate samples. p61-Sema3E signaling enhanced the transendothelial migration of tumor cells, whereas this effect was lost in Plexin D1-deficient cells. ${ }^{* *} P<0.0005$.

not been elucidated. Notably, a common feature of guidance cues, including Sema3E, is their ability to act as double-faced signals. Here we found that Sema3E is able to independently regulate endothelial and tumor cells, triggering divergent functional outcomes, through the involvement of distinctive signaling pathways. When we asked about the functional relevance of Sema3E signaling in tumor development and metastatic progression, we found that Sema3E endogenously expressed in tumor cells is a major determinant of their invasive-metastatic behavior in vivo. Moreover, and relevant to the clinics, we found that Sema3E expression in human primary tumors correlates with their potential to form metastasis.

Sema3E had been initially identified in cancer cells; however, it was unclear whether its expression could impinge on tumor growth. We found that Sema3E overexpression reduced tumor burden in vivo. According to our data, this was not accounted for by direct suppression of tumor cell growth. In fact, the ectopic expression of Sema3E in different cancer cells did not inhibit their proliferation or survival in culture, including upon anchorage-independent growth in soft agar (A. Casazza and L. Tamagnone, unpublished observa- tions). Moreover, while apoptosis was increased in Sema3E-overexpressing tumors, the proliferation index of cancer cells was not significantly reduced. In fact, the tumor suppressor effect observed in our experiments was likely accounted for by the reduced vascular density induced by sustained paracrine signaling of ectopic Sema3E released in the tumor microenvironment, consistent with the endothelial repelling function observed in developmental angiogenesis (20). Moreover, we elucidated that the endothelial-repelling and inhibitory activity of Sema3E depends on the R-Ras GAP activity of Plexin D1 and on the expression of the regulatory molecule Rnd2. It is noteworthy that the potential inhibitory activity of endogenous Sema3E levels released by tumor cells is probably overpowered in vivo by proangiogenic factors in the tumor microenvironment. Consistent with this hypothesis, we found that interfering with endogenous Sema3E expression in cancer cells did not increase tumor growth, while it significantly curbed metastatic spreading.

We have reported previously that Sema3E undergoes proteolytic cleavage by furin-like PPCs. This maturation process is strictly required to unleash the prometastatic activity of the semaphorin, 
A

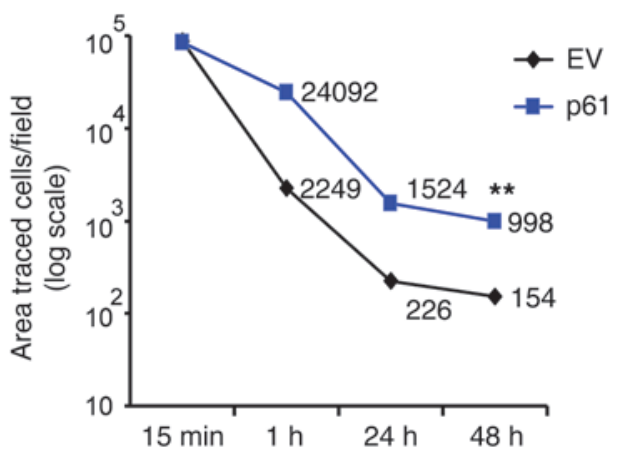

B

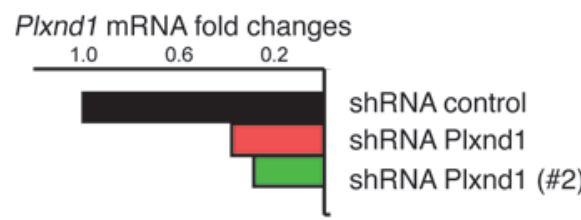

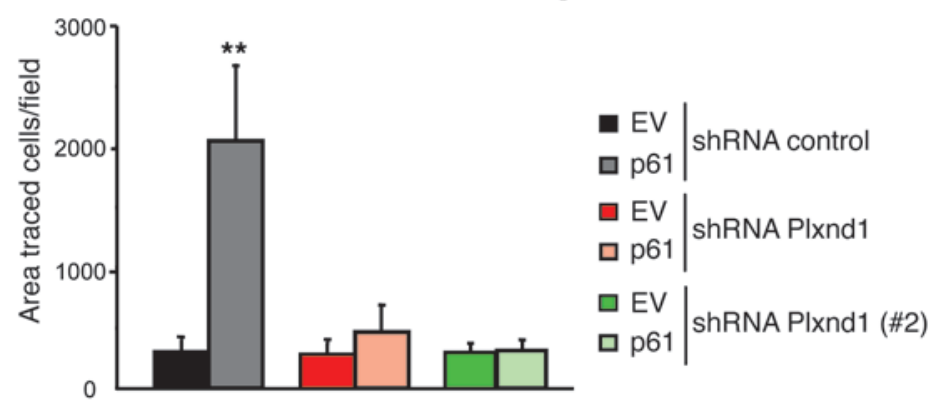

Figure 7

p61-Sema3E enhances cancer cell metastatic extravasation. (A) MDA-MB-435 cancer cells expressing p61 (as in Figure 6) were fluorescently labeled and injected intravenously in nude mice. Two days later, the number of metastatic fluorescent cells was quantified in 4 lungs for each experimental group (at least 10 independent stereomicroscopic fields each) with METAMORPH software. Data are given as the mean of traced cells per field. ${ }^{* \star} P<0.005$. (B) The same engineered MDA-MB-435 cancer cells shown in Figure 6D, plus 2 additional batches of Plexin D1depleted cells (expressing an alternative shRNA sequence indicated by "\#2"; see RT-PCR analysis on top) were fluorescently labeled and injected intravenously in nude mice. The number of fluorescent cells in the lungs was quantified by analyzing 4 mice for each experimental group (at least 10 independent stereomicroscopic fields each) with METAMORPH software. Data are given as mean of traced cells per field \pm SD. The $y$-axis is in logarithmic scale. ${ }^{* *} P<0.005$.

since a mutated uncleavable form of full-length Sema3E has no prometastatic activity (19). In fact, PPCs are commonly expressed in tumor cell lines and human cancers, and our present data indicate that the small proteolytic fragment p61 is the main form of Sema3E found in the conditioned media of tumor cells and in experimental tumor samples. Importantly, we have shown here that the p61 fragment is fully competent to bind the functional receptor Plexin D1, and it carries an endothelial cell-repelling activity comparable with that of the full-length molecule.

In apparent contrast with its potential inhibitory function in endothelial cells, p61-Sema3E enhances the inherent invasiveness of tumor cells and their metastatic behavior. Moreover, the receptor Plexin D1 is responsible for this autocrine effect, since knocking down its expression in tumor cells abates their metastatic potential and abrogates any proinvasive and metastatic response to p61. Metastasis formation is a multistep process, starting with tumor cell invasiveness at the primary tumor site and implicating cell migration, cell survival, and matrix degradation as well as the ability to overcome tissue barriers, such as vessel walls, and ultimately leading to colonization of distant tissues (33). In our study, we found that p61-Sema3E promotes tumor cell migration and invasiveness, possibly mediated by invadopodia formation. Notably, on the basis of multiple functional experiments in vitro (including adhesion-independent growth and survival in soft agar), we have ruled out that p61 may exert an antiapoptotic activity in tumor cells, potentially impinging on their survival in the blood stream (A. Casazza and L. Tamagnone, unpublished observations). Instead, we found that the Sema3E expression levels dictate the ability of circulating cancer cells to extravasate and form metastatic colonies.

A few other semaphorins have been found to promote cell migration by transactivating plexin-associated tyrosine kinase receptors in a cell-specific manner (4-6). This suggested that an RTK could be similarly involved in Sema3E signaling, especially since we had observed Plexin D1 phosphorylation on tyrosine upon p61 stimulation. Primed by the results of a phospho-proteomic screening, we then demonstrated that ErbB2 is the tyrosine kinase receptor responsible for p61-mediated signaling, leading to the invasivemetastatic response. Notably, both the treatment with an ErbB2 inhibitor and the selective knockdown of this RTK by RNAi resulted in a dramatic reduction of p61-induced tumor cell migration and metastatic lung colonization.

ErbB2 tyrosine kinase receptor is commonly expressed in human tumors, but it has no cognate ligand, and it mainly becomes activated by transphosphorylation in association with the homologous receptors EGFR or ErbB3 (30-32). We observed a minor increase of EGFR but not ErbB3 phosphorylation in tumor cells treated with p61-Sema3E, possibly suggesting that Plexin D1 signalling impinges on the activation of ErbB2-EGFR receptor heterodimers found in cancer cells but not in endothelial cells (see our preliminary data in Supplemental Figure 13, A and B). Moreover, MAPK and PLC- $\gamma$ appeared to be the main signal transducers involved in the pathway, while PI3K-AKT activation was only marginally implicated. This is consistent with that reported in literature about the predominant involvement of PLC- $\gamma$ and MAPK pathways downstream to ErbB2-EGFR complexes $(30,31)$.

It has been shown in neurons that neuropilin-1 expression levels can dictate differential functional responses to Sema3E in different cell populations (26). Intriguingly, neuropilin-1 is expressed in HUVECs at an intermediate level between those found in the 2 tumor cell models used in our functional experiments in vivo, i.e., MDA-MB-435 and A549 cells (data not shown). Moreover, p61Sema3E cannot interact with neuropilin-1 (data not shown). Thus, our data do not support the idea that neuropilin expression alone may account for the functional differences between Sema3E activity in endothelial and tumor cells. Interestingly, we found that the small 
A

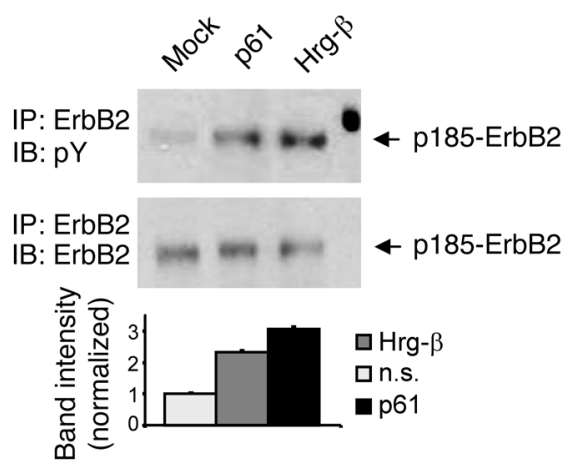

B

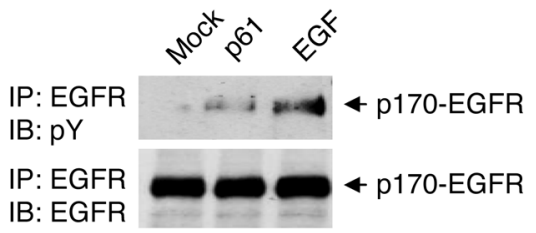

IP: ErbB3

IB: $p Y$

IP: ErbB3

IB: ErbB3

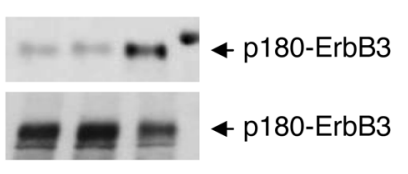

C
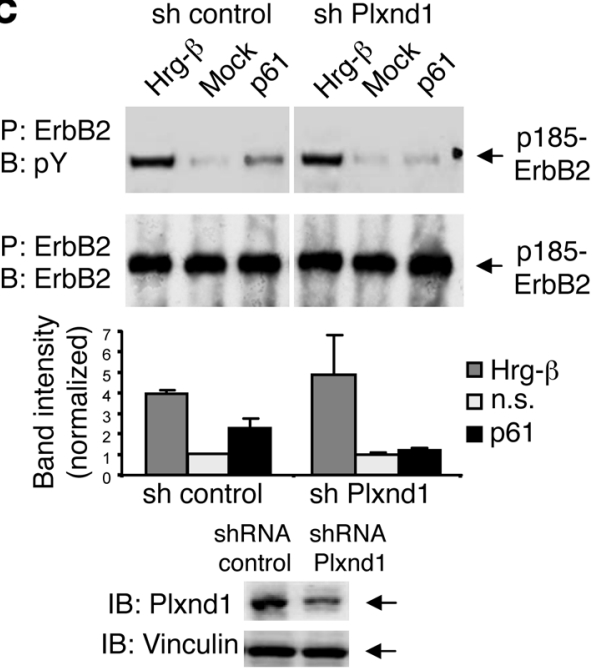

$\mathbf{F}$

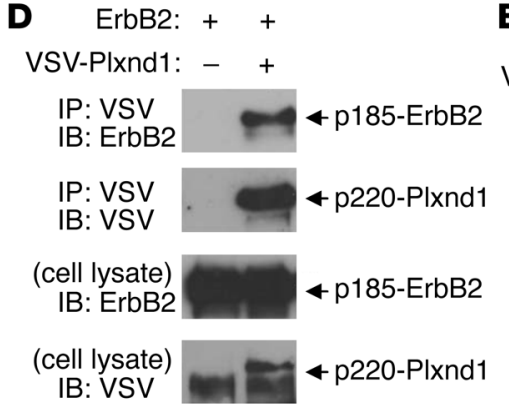

\section{$\mathbf{E}$}

$\begin{array}{rllllll}\text { VSV-PIxnd1: } & - & + & + & + & + & + \\ \text { p61: } & + & - & - & + & + & + \\ \text { Lapatinib: } & + & - & + & - & - & + \\ \text { PHA: } & - & - & - & - & + & -\end{array}$

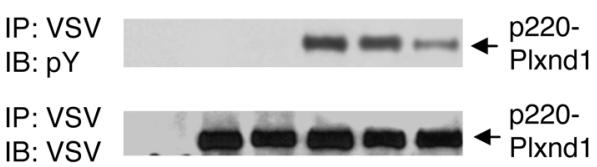

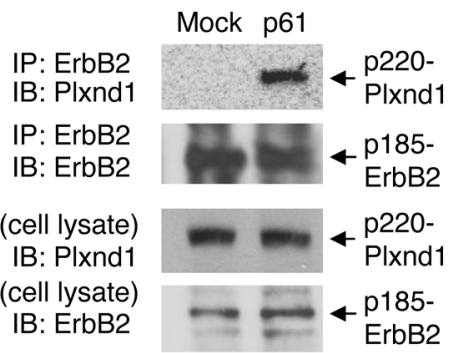

Figure 8

p61-Sema3E elicits the activation of Plexin D1-associated tyrosine kinase ErbB2. (A) A549 tumor cells were starved for 48 hours and stimulated for 15 minutes with $7 \mathrm{nM}$ p61-Sema3E or $0.2 \mathrm{nM}$ Heregulin- $\beta 1 \mathrm{ECD}(\mathrm{Hrg}-\beta)$. ErbB2 was immunoprecipitated and analyzed by immunoblotting with anti-phospho-tyrosine (anti-pY) antibodies. The graph shows the average fold change of band intensity \pm SD observed in 3 experiments (normalized to controls). (B) As above, serum-starved A549 cells were stimulated for 15 minutes with 7 nM p61 or 1 nM EGF. Then, EGFR or ErbB3 were immunoprecipitated using appropriate antibodies and analyzed with anti-phospho-tyrosine antibodies. (C) In analogy to the above experiments, ErbB2 phosphorylation in response to $\mathrm{p} 61$ or Hrg- $\beta$ was assayed in HeLa carcinoma cells, either control or Plexin D1 depleted (verified by Western blot analysis). The graph shows the average fold change of band intensity \pm SD observed in 3 experiments. (D) Plexin D1 (VSV-tagged) and ErbB2, transfected into HEK293 cells, coprecipitate in a specific complex, as revealed by immunoblotting. Analogous results were obtained upon transfection in COS cells (data not shown). The experiment was repeated 3 times with consistent results. (E) COS cells transfected with VSV-tagged Plexin D1 were preincubated with either $200 \mathrm{nM}$ Lapatinib, or $250 \mathrm{nM}$ PHA-665752 (PHA), or vehicle, for 3 hours. Thereafter, cells were treated for 15 minutes with $7 \mathrm{nM} \mathrm{p61}$ or mock stimulated. Plexin D1 was immunoprecipitated and analyzed with anti-phospho-tyrosine antibodies. (F) Serum-starved A549 cancer cells, expressing endogenous Plexin D1 and ErbB2 receptors, were treated with mock or $7 \mathrm{nM}$ p61-Sema3E for 10 minutes. Plexin D1 copurifying with ErbB2 was revealed by immunoblotting.

GTPase Rnd2, associated with Plexin D1, is required for the inhibitory activity of Sema3E in endothelial cells while it is expressed at low levels and not implicated in Sema3E function in tumor cells (see Supplemental Figure 10, A and B). Conversely, tumor cells are characterized by the expression of ErbB2-EGFR heterodimers, which are involved in Sema3E-induced invasion and metastasis.

Previously published data on the expression of Sema3E and its receptor in human tumors do not univocally establish a correlation with tumor progression. For instance, we have shown high Sema3E levels in mammary carcinomas (19). Moreover, others have found an elevated expression in high- versus low-grade glioblastomas (34), while the opposite was true for bladder carcinomas (35). As expected, the functional relevance of Sema3E as a master regulator of the invasive-metastatic phenotype may depend on the activity of additional tumor type-specific regulatory pathways as well as on individual genetic variability. Of note, Roodink and coworkers, in an immunohistochemistry analysis of nevi and melanoma samples, came to the puzzling conclusion that while Plexin D1 expression is positively correlated with tumor metastatic progression, Sema3E levels are regulated in the opposite manner (28). We have now analyzed a wide panel of nevi and melanoma samples, using a validated antibody proven to detect all isoforms of Sema3E, including p61 (19). Our data demonstrate a significant increase of Sema3E expression in highly invasive and metastatic melanomas, compared with that of benign nevi and noninvasive melanomas. Notably, our data are further consistent with those seen in 2 independent gene expression data sets $(36,37)$ and support the postulated role of Sema3E signaling in the invasive-metastatic progression of melanomas.

We have furthermore analyzed the expression of Sema3E and its receptor in a wide series of colon cancer samples, for which we obtained detailed clinicopathological information. We found that while Sema3E expression is often below the detection threshold in 
A

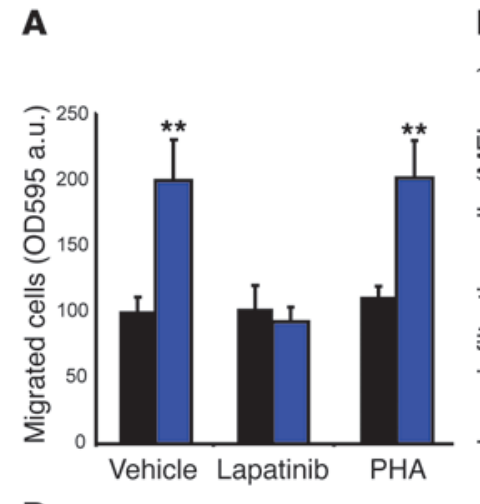

D

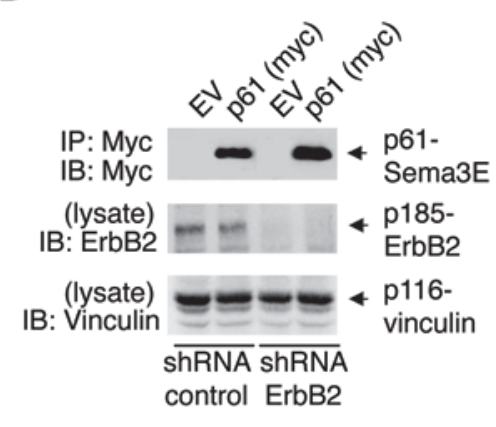

B
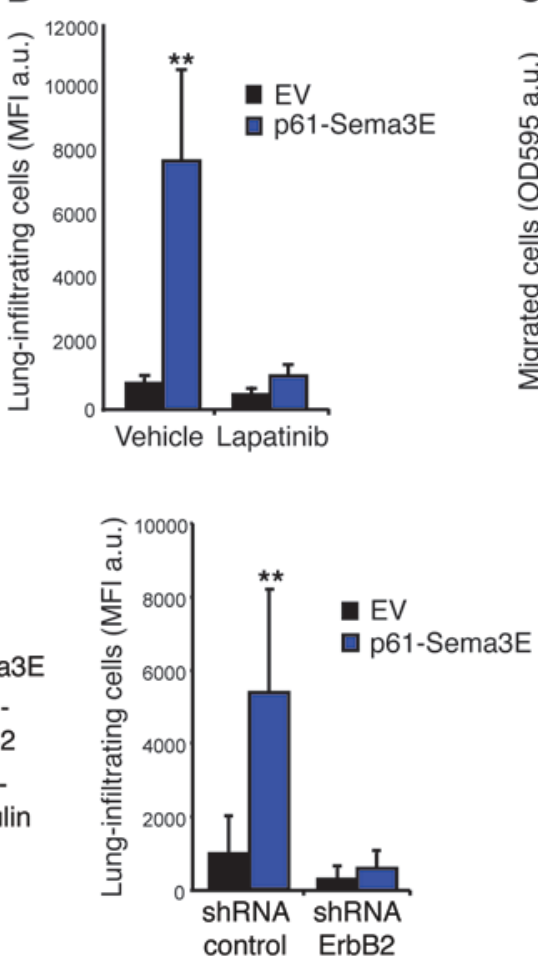

C

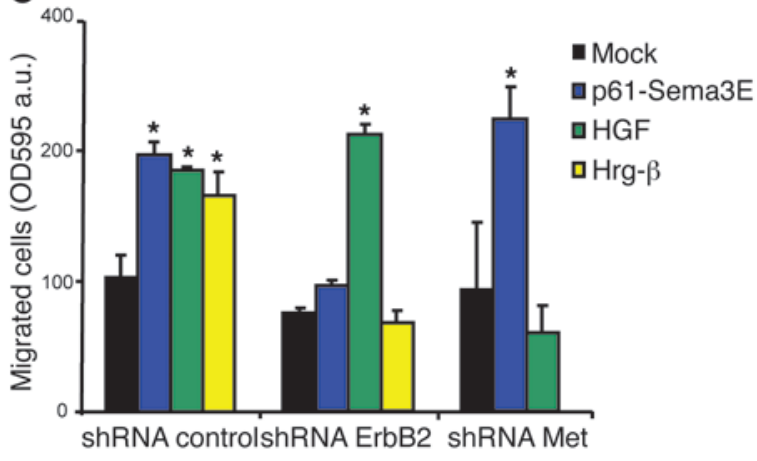

E

Figure 9

ErbB2 kinase is required for p61-dependent promigratory and prometastatic function. (A) A549 cells were pretreated with $400 \mathrm{nM}$ Lapatinib or $250 \mathrm{nM}$ PHA-665752 for 2 hours and allowed to migrate in a Transwell insert in response to $7 \mathrm{nM} \mathrm{p61-Sema3E}$ in presence of the same inhibitors. Migrated cells were quantified by staining with crystal violet (see Methods). Data are given as average \pm SD of 2 independent experiments. ${ }^{* *} P<0.005$. Analogous results were obtained by analyzing MDA-MB-435 and HeLa cancer cells (see Supplemental Figure 11, A and B). (B) A549 cells were transduced to establish an autocrine circuit of p61-Sema3E, fluorescence labeled with CFDA-SE, and injected systemically into mice treated with Lapatinib or vehicle (see Methods). Forty-eight hours after injection, metastatic fluorescent cells in the lungs were quantified (as described in Methods). The graph indicates average values \pm SD of 5 mice per each experimental group. ${ }^{* *} P<0.003$. (C) The expression of ErbB2 (or Met tyrosine kinase as control) was knocked down in A549 tumor cells by RNAi (see Methods) and migration was assayed as above in response to $7 \mathrm{nM}$ p61, $1 \mathrm{nM} \mathrm{Hrg}-\beta$, or $1 \mathrm{nM} \mathrm{HGF}$. Data are given as average \pm SD of 2 independent experiments. Statistical significance was calculated relative to mocktreated cells in each group. ${ }^{*} P<0.05$. (D) ErbB2-depleted A549 cells and respective controls were transduced to establish an autocrine circuit of p61-Sema3E (see expression analysis). Tumor cells were labeled with CFDA-SE before intravenous injection into nude mice. The graph shows the number of metastatic cells infiltrating the lungs after 48 hours, quantified as in B (average values \pm SD of 5 mice per each experimental group). ${ }^{\star \star} P<0.005$. (E) Sema3E-induced migration of A549 cells was assayed as above, in the presence of DMSO (vehicle), or $2 \mu \mathrm{m}$ U73122 (PLC $\gamma$ ), $10 \mu \mathrm{m}$ PD98059 ("PD," MAPK inhibitor), or $10 \mu \mathrm{m}$ LY294002 ("LY," PI3K inhibitor). PLC $\gamma$ close to U73122; (MAPK inhibitor) close to PD98059, and (AKT inhibitor) close to LY294002. Data are given as average \pm SD of 2 independent experiments. ${ }^{*} P<0.05,{ }^{* *} P<0.003$.

nonmetastatic tumors, it is almost invariably present in primary tumors, giving rise to distant metastasis. Moreover, the average expression levels of both Sema3E and Plexin D1 were significantly increased in excised metastases compared with those of primary tumors, suggesting the acquisition of a selective advantage for metastatic spreading in cell-autonomous manner. This was confirmed upon analyzing gene expression in a subset of 8 primary tumors with available matched metastatic tissue from the same patients. In fact, the levels of both Plexin D1 and Sema3E were further increased in the metastasis compared with the primary tumor in $100 \%$ and $75 \%$ of these cases, respectively (Supplemental Figure $1 \mathrm{~B})$. Moreover, the median expression value had a more than 6 -fold increase in metastases for both genes, and this difference passed statistical significance tests for Plexin D1 $(P=0.004)$.

Considering our data on the involvement of ErbB2 in p61Sema3E signaling, one would expect that metastatic human tumors expressing Sema3E and Plexin D1 should also contain
ErbB2. We clearly detected ErbB2 expression in all primary colon carcinoma samples included in our panel (average of transcripts per $10^{4}$ copies $\beta$-actin, $15.32 \pm 2.57 \pm \mathrm{SEM}$ ). However, there was no significant statistical correlation between ErbB2 expression levels and the metastatic or nonmetastatic behavior of these tumors (data not shown). This indicates that, not unexpectedly, the ErbB2 oncogene is not the driving force for the metastatic spreading of colon cancers, but it is widely expressed in tumors, consistent with an effector function in Sema3E-Plexin D1 signaling cascade.

We finally asked whether Sema3E expression could have any relevance as a predictor of clinical outcome in human cancers. Although providing a conclusive answer to this question is beyond the scope of this study, we looked carefully into our data on colon cancer patients. As expected, while early stage tumors (i.e., T1-T2) did not associate with metastasis, advanced stage colon cancers (i.e., T3-T4) had an overall $28 \%$ incidence of metastasis in our series (12 out of 43 cases). However, we noticed that among Sema3E- 
negative tumors the incidence of metastasis was only $10 \%$ (2 out of 19), whereas Sema3E-positive primary tumors had a $58 \%$ incidence of metastasis (14 out of 24). Although the size of this sample is small, Fisher's exact test confirmed that this is not a random distribution $(P=0.03)$, suggesting that Sema3E expression may predict the risk of metastatic progression in advanced tumors. To further assess the clinical significance of Sema3E expression in primary colorectal tumors, we analyzed a subset of patients for which the clinical outcome after 2 years of follow-up was known (Supplemental Figure 1C). Interestingly, Kaplan-Meier analysis revealed that Sema3E levels in primary tumors inversely correlated significantly with patient survival. Indeed, the overall 2-year survival rate was $94 \%$ in patients with Sema3E-negative primary tumors but only $58 \%$ in patients with Sema3E-positive tumors $(P=0.02$ by log-rank test; $n=16$ for Sema3E-negative tumors and $n=12$ for Sema3E-positive tumors). Thus, our data strongly suggest that Sema3E expression and signaling (via Plexin D1) correlate positively with metastatic progression and poor prognosis of human colon carcinomas. Moreover, taken together, our findings indicate Sema3E as an important "modifier" signal in cancer progression, potentially active in multiple human tumors.

In conclusion, we showed that Plexin D1 stimulation by p61Sema3E can elicit 2 independent signaling cascades and divergent functional effects in cancer cells and in endothelial cells. Moreover, we elucidated the functional relevance in vivo of Sema3E and Plexin D1 endogenously expressed in tumor cells, by showing that their partial knockdown is sufficient to abate the metastatic potential, while we ruled out that blocking endogenous Sema3E might promote tumor growth. We have furthermore provided statistical evidence in colon cancer patients that an elevated expression of Sema3E (and Plexin D1) strikingly increases the risk of metastatic progression. In sum, Sema3E autocrine signaling is crucially implicated to induce a metastatic behavior in multiple cancer cells, and it appears as a promising target for strategies aimed at blocking tumor metastasis.

\section{Methods}

Further information can be found in the Supplemental Methods.

Antibodies and other reagents. Myc-tagged and VSV-tagged proteins were detected by MoAb 9E10 (Santa Cruz Biotechnology Inc.) and P5D4 (Sigma-Aldrich), respectively. Other antibodies were as follows: anti-mouse CD31 (MEC 13.3, BD Biosciences), anti-Ki-67 (DAKO), anti-activated caspase-3 (Cell Signaling Technology), anti-endoglin (R\&D Systems), antiphosphotyrosine (4G10, Upstate), biotinylated anti-human Plexin D1 (aa 47-1271, R\&D Systems), anti-ErbB2 (29D8, Cell Signaling Technology, and e2-4000, Invitrogen), anti-EGFR (LA22, Millipore), anti-ErbB3 (C-17, Santa Cruz Biotechnology Inc.), anti-Rnd2 (Proteintech Group Inc.), antiCaspase-9 (96.1.23, Santa Cruz Biotechnology Inc.), anti-laminB (101-B7, Calbiochem), anti-actin (C-11, Santa Cruz Biotechnology Inc.), anti-HA (Y-11, Santa Cruz Biotechnology Inc.), and anti-Vinculin (V4505, SigmaAldrich). Antibodies to Sema3E were described previously (19). Recombinant human Heregulin- $\beta 1$ Extracellular Domain (Hrg- $\beta$ ) and HGF were from R\&D Systems. Human recombinant EGF was from Sigma-Aldrich. Lapatinib (GW572016, Tykerb) was provided by GlaxoSmithKline or purchased from Sellek Chemicals. Met inhibitor PHA665752 was from TOCRIS Bioscience. LY294002 and PD98059 were from Calbiochem, and U-73122 was from Sigma-Aldrich.

Ectopic gene expression in mammalian cells. Expression constructs encoding full-size mouse Sema3E (fused with a polyHis-Myc-epitope tag at C'-term) as well as truncated p61-Sema3E (19) were subcloned into the lentiviral transfer plasmid pRRLsin.cPPT.hCMV.GFP.Wpre, and gene transfer was performed as previously described (38). This method ensured stable gene transfer with very high efficiency, without the need to select individual cell clones. In addition, we assayed multiple independent batches of cells transduced with each construct. The ErbB2 mammalian expression construct was a gift from Thomas Diettmar (Witten/Herdecke University, Witten, Germany). A dominant-active R-Ras was provided by Dominique T. Brandt (University of Heidelberg, Heidelberg, Germany). An expression construct for Rnd2 was purchased from Missouri S\&T cDNA Resource Center.

Purification of Sema3E molecules. COS cells were transduced to stably express the respective Sema3E molecules. Conditioned media were harvested from these cells in the absence of serum. Protein purification was achieved using a Nickel-loaded affinity column (GE-Pharmacia) according to the manufacturer's protocols.

Knockdown of gene expression by RNAi. The expression of Plexin D1, Sema3E, ErbB2, and Met was silenced in tumor and/or endothelial cells by expressing gene-targeted shRNA. Rnd2 expression was knocked down in HUVECs by transfection with targeted siRNA duplexes. Details on reagents, procedures, and the targeted sequences are provided in the Supplemental Methods.

Real-time quantitative PCR analysis of gene expression. Total RNA from tumor cell lines or tissues was isolated by using RNeasy Protect Mini Kit (Qiagen) according to the manufacturer's instructions. cDNA preparation was performed according to standard procedures, using M-MLV Reverse Transcriptase (Promega) and oligo-dT primers (Sigma-Aldrich). Real-time quantitative PCR (Q-PCR) analysis was performed using the Applied Biosystems 7900 HT Fast Real-Time PCR System and PCR primers and TaqMan probes listed in Supplemental Methods. Gene expression in cells subjected to RNAi-mediated knockdown was quantified as relative levels versus mock-transduced cells. On the other hand, gene expression in individual human tumor samples was quantified as relative number of transcripts compared with $\beta$-actin. Informed consent was received for human samples. Care and use of human samples were approved by review boards at the University of Heidelberg (Germany), and the Institute for Cancer Research and Treatment (Candiolo, Turin, Italy).

MTT cell viability assay. Transduced tumor cells were seeded in multiple 24 -well plates $\left(5 \times 10^{4}\right.$ cells/well). After 24 hours of serum starvation, the cells were cultured in DMEM medium containing 0.1\% FBS. To reveal viability, the cells were incubated with $500 \mu \mathrm{l} / \mathrm{ml}$ of 3-(4,5-dimethylthiazol-2-yl)-2,5-diphenyltetrazolium bromide (MTT) dye solution (Sigma-Aldrich) for 4 hours at $37^{\circ} \mathrm{C}$. The indicator product, formazan, was resuspended in DMSO and measured spectrophotometrically at $570 \mathrm{~nm}$ by a Titertek Multiskan reader.

Fluorescence labeling of living cells. MDA-MB-435 cells were incubated with $10 \mu \mathrm{M}$ CFDA SE Vybrant Cell Tracer (Molecular Probes) for 15 minutes at $37^{\circ} \mathrm{C}$. Then the loading solution was replaced with fresh, prewarmed medium and the cultures were incubated for another 30 minutes at $37^{\circ} \mathrm{C}$.

Cell migration and invasion assays. Cell motility was assayed using Transwell chamber inserts (Costar), with a porous polycarbonate membrane $(8-\mu \mathrm{M}$ pore size) as described previously (39). To allow haptotactic migration, the lower side of the filter was coated with $10 \mu \mathrm{g} / \mathrm{ml}$ fibronectin. Cells were harvested from culture dishes by treatment with $1 \mathrm{mM}$ EDTA and resuspended in $0.2 \%$ BSA-containing medium. Approximately $1 \times 10^{5}$ cells were added in the upper chamber and allowed to migrate through the filter toward the lower chamber (including regulatory molecules, when indicated) for 6-8 hours in a cell culture incubator. Then, the cells adherent to the upper side of the filter were mechanically removed, while those that migrated to the lower side of the filter were fixed with $11 \%$ glutaraldehyde and stained with crystal violet. The dye was then solubilized in $10 \%$ acetic acid to measure absorbance at $595 \mathrm{~nm}$ in a microplate reader (OD values are shown in the graphs as arbitrary units). For ECM invasion assays, $5 \times 10^{5}$ tumor cells 
were added to the upper chamber of a Transwell insert coated with Matrigel $(20 \mu \mathrm{g} /$ well; Becton Dickinson) and allowed to migrate in 1\% FBS-containing medium. After 16 hours of incubation, the cells adherent to the lower side of the porous membrane were fixed and analyzed as above.

Transendothelial migration of tumor cells. Approximately $1 \times 10^{5}$ HUVECs were added to type I collagen-coated 24-well Transwell inserts with small pore sizes $(5-\mu \mathrm{m}$ pore size; Costar) and grown to confluence for 3 days, with daily replacement of fresh culture medium, until they formed a tightly packed monolayer. Then, $7 \times 10^{5}$ CFDA-SE-labeled (see above) MDAMB-435 cells, resuspended in HUVEC culture medium, were added to the upper chamber. After incubation for 16 hours, the cells were fixed with $11 \%$ glutaraldehyde, and the upper side of the membranes were gently scraped with a cotton swab to remove nonmigrated cells. Transmigrated tumor cells were quantified based on green fluorescence in 5 random fields, using METAMORPH software.

Animal model and in vivo procedures. In vivo studies were conducted in 6- to 8-week-old immunocompromised $\mathrm{Cd} 1^{-/-}$nude athymic or BALB/C female mice (Charles River Laboratory). Unless otherwise indicated, in each experiment, we analyzed 6 tumors per each condition. For subcutaneous tumor models, $2 \times 10^{6}$ MDA-MB-435, $8 \times 10^{6}$ A549, or $5 \times 10^{5} 4 \mathrm{~T} 1$ cells were injected into the right posterior flank of anesthetized animals. Tumor size was measured externally every 2 days using a caliper, and tumor volume was estimated using the equation $V=4 / 3 \pi \times(d / 2)^{2} \times D / 2$, where $d$ indicates the minor tumor axis, $D$ indicates the major tumor axis, and $V$ indicates volume. Mice were sacrificed after 30-60 days after transplant, and tumors were weighted after dissection. Superficial pulmonary metastases were contrasted using black India ink to infuse airways, and counted on dissected lung lobes under a stereoscopic microscope. For orthotopic injection, $1 \times 10^{6} 4 \mathrm{~T} 1$ cells resuspended in $40 \mu \mathrm{l}$ phosphate-buffered saline were injected into the second mammary fat pad of anesthetized Balb/c mice, following exposure by a small incision. Mice were sacrificed after 25 days, and tumors and lungs were measured as mentioned above. For experimental metastasis assays, $2 \times 10^{6}$ MDA-MB-435, $6 \times 10^{6}$ A549, or $2 \times 10^{5} 4 \mathrm{~T} 1$ cells were injected into the lateral mouse tail vein; for long-term experiments, cell colonies were scored in the lung using black India ink to infuse airways, as described above. Short-term metastatic extravasation of fluorescent cancer cells in the lungs was quantified by analyzing 4 pairs of lungs for each experimental group (at least 10 independent microscopic fields each) with METAMORPH software. For experiments based on in vivo Lapatinib treatments, mice received the vehicle $(0.5 \%$ hydroxypropylmethylcellulose with $0.1 \%$ Tween 80 in water) or the drug (100 mg/ $\mathrm{kg}$ body weight) daily by oral gavage, from 3 days before to 2 days after systemic tumor cell injection. Animal procedures had been approved by the Ethical Commission of the University of Torino and by the Italian Ministry of Health.
Histological analysis. Paraffin-embedded or OCT-embedded frozen samples were cut in $10-\mu \mathrm{m}$ thick sections and probed with primary antibodies (listed above). Streptavidin was conjugated with HRP (DAKO), while all secondary antibodies used were conjugated with Alexa Fluor 488 or Alexa Fluor 546 fluorochromes (Molecular Probes). To detect apoptotic cells in tumor sections, we used an indirect TUNEL assay, using the ApopTag Green In Situ Apoptosis Detection Kit (Chemicon). Slides were analyzed using a Leica DM IRBM microscope, and digital images were evaluated with METAMORPH software. Unless otherwise indicated, quantification was done by analyzing at least 3 sections and 10 fields per tumor (considering 6 tumors per experimental condition).

Immunoprecipitation, SDS-PAGE, and Western immunoblotting. Cellular proteins were analyzed using standard methods, as described in the Supplemental Methods, using antibodies listed above. Band intensity quantification was done with Quantity-One software.

Statistics. Statistical significance was verified using the 2-tailed Student's $t$ test. $P$ values of less than 0.05 were considered significant.

\section{Acknowledgments}

We gratefully acknowledge the help of E. Vigna, S. Corso, and S. Giordano (IRCC, Candiolo) and T. Diettmar (Witten/Herdecke University, Germany) and D.T. Brandt (University of Heidelberg, Germany) for generously sharing expression constructs important for this study. We thank E. Urbano, L. Palmas, and F. Sassi for skillful technical assistance; M. Galluzzo, F. Maina, T. Werdiningsih, and L. Tarditi for support with experiments in mice; F. Galimi for help with Q-PCR analysis; and S. Giordano, G. Neufeld, and C. Isella for useful discussion and suggestions. The work was supported by grants from Italian Association for Cancer Research and from Regione Piemonte (to L. Tamagnone, L. Trusolino, and P.M. Comoglio). A. Casazza is supported by a FIRC fellowship.

Received for publication December 21, 2009, and accepted in revised form May 19, 2010.

Address correspondence to: Luca Tamagnone, IRCC, S.P. 142, 10060, Candiolo (Torino), Italy. Phone: 39.011.9933.204; Fax: 39.011.9933.225; E-mail: luca.tamagnone@ircc.it. Or to: Massimiliano Mazzone, VIB Leuven, Belgium. Phone: 32.16.34.61.76; Fax: 32.16.34.59.90; E-mail: massimiliano.mazzone@vib-kuleuven.be.

Charlotte Rolny's present address is: University of Uppsala, Department of Genetics and Pathology, Vascular Biology Unit, Rudbeck Laboratory, Uppsala, Sweden.
1. Zhou Y, Gunput RA, Pasterkamp RJ. Semaphorin signaling: progress made and promises ahead. Trends Biochem Sci. 2008;33(4):161-170.

2. Tamagnone L, Comoglio PM. Signalling by semaphorin receptors: cell guidance and beyond. Trends Cell Biol. 2000;10(9):377-383.

3. Casazza A, Fazzari P, Tamagnone L. Semaphorin signals in cell adhesion and cell migration: functional role and molecular mechanisms. Adv Exp Med Biol. 2007;600:90-108.

4. Giordano S, et al. The semaphorin $4 \mathrm{D}$ receptor controls invasive growth by coupling with Met. Nat Cell Biol. 2002;4(9):720-724.

5. Toyofuku T, et al. Dual roles of Sema6D in cardiac morphogenesis through region-specific association of its receptor, Plexin-A1, with off-track and vascular endothelial growth factor receptor type 2 . Genes Dev. 2004;18(4):435-447.

6. Swiercz JM, Kuner R, Offermanns S. Plexin-B1 RhoGEF-mediated RhoA activation involves the receptor tyrosine kinase ErbB-2. J Cell Biol. 2004;165(6):869-880.

7. Swiercz JM, Worzfeld T, Offermanns S. ErbB-2 and Met reciprocally regulate cellular signaling via Plexin-B1. J Biol Chem. 2008;283(4):1893-1901.

8. Franco M, Tamagnone L. Tyrosine phosphorylation in semaphorin signalling: shifting into overdrive. EMBO Rep. 2008;9(9):865-871.

9. Neufeld G, Kessler O. The semaphorins: versatile regulators of tumour progression and tumour angiogenesis. Nat Rev Cancer. 2008;8(8):632-645.

10. Capparuccia L, Tamagnone L. Semaphorin signaling in cancer cells and in cells of the tumor microenvironment--two sides of a coin. J Cell Sci. 2009;122(pt 11):1723-1736

11. Castro-Rivera E, Ran S, Thorpe P, Minna JD. Semaphorin 3B (SEMA3B) induces apoptosis in lung and breast cancer, whereas VEGF165 antagonizes this effect. Proc Natl Acad Sci U S A. 2004;101(31):11432-11437.
12. Rolny $\mathrm{C}$, et al. The tumor suppressor semaphorin $3 \mathrm{~B}$ triggers a prometastatic program mediated by interleukin 8 and the tumor microenvironment. J Exp Med. 2008;205(5):1155-1171.

13. Bielenberg DR, et al. Semaphorin 3F, a chemorepulsant for endothelial cells, induces a poorly vascularized, encapsulated, nonmetastatic tumor phenotype. J Clin Invest. 2004;114(9):1260-1271.

14. Kessler O, et al. Semaphorin-3F is an inhibitor of tumor angiogenesis. Cancer Res. 2004; 64(3):1008-1015.

15. Basile JR, Castilho RM, Williams VP, Gutkind JS. Semaphorin 4D provides a link between axon guidance processes and tumor-induced angiogenesis. Proc Natl Acad Sci U S A. 2006;103(24):9017-9022.

16. Sierra JR, et al. Tumor angiogenesis and progression are enhanced by Sema4D produced by tumor-associated macrophages. J Exp Med. 2008;205(7):1673-1685.

17. Christensen CR, Klingelhöfer J, Tarabykina S, Hul- 
gaard EF, Kramerov D, Lukanidin E. Transcription of a novel mouse semaphorin gene, $\mathrm{M}$-semaH, correlates with the metastatic ability of mouse tumor cell lines. Cancer Res. 1998;58(6):1238-1244.

18. Martín-Satué M, Blanco J. Identification of semaphorin E gene expression in metastatic human lung adenocarcinoma cells by mRNA differential display. J Surg Oncol. 1999;72(1):18-23.

19. Christensen C, et al. Proteolytic processing converts the repelling signal Sema3E into an inducer of invasive growth and lung metastasis. Cancer Res. 2005;65(14):6167-6177.

20. Gu C, et al. Semaphorin 3E and plexin-D1 control vascular pattern independently of neuropilins. Science. 2005;307(5707):265-268.

21. Gitler AD, Lu MM, Epstein JA. PlexinD1 and semaphorin signaling are required in endothelial cells for cardiovascular development. Dev Cell. 2004;7(1):107-116.

22. Moriya J, et al. Inhibition of semaphorin as a novel strategy for therapeutic angiogenesis. Circ Res. 2010;106(2):391-398.

23. Roodink I, et al. Plexin D1 expression is induced on tumor vasculature and tumor cells: a novel target for diagnosis and therapy? Cancer Res. 2005;65(18):8317-8323.

24. Roodink I, Verrijp K, Raats J, Leenders WP. Plexin D1 is ubiquitously expressed on tumor vessels and tumor cells in solid malignancies. BMC Cancer.
2009;9:297.

25. Uesugi K, Oinuma I, Katoh H, Negishi M. Different Requirement for Rnd GTPases of R-Ras GAP Activity of Plexin-C1 and Plexin-D1. J Biol Chem. 2009;284(11):6743-6751.

26. Chauvet S, et al. Gating of Sema3E/PlexinD1 signaling by neuropilin-1 switches axonal repulsion to attraction during brain development. Neuron. 2007;56(5):807-822.

27. Kigel B, Varshavsky A, Kessler O, Neufeld G. Suc cessful inhibition of tumor development by specific class- 3 semaphorins is associated with expression of appropriate semaphorin receptors by tumor cells. PLoS ONE. 2008;3(9):e3287.

28. Roodink I, et al. Semaphorin 3E expression correlates inversely with Plexin D1 during tumor progression. Am J Pathol. 2008;173(6):1873-1881.

29. Yilmaz M, Christofori G. EMT, the cytoskeleton, and cancer cell invasion. Cancer Metastasis Rev. 2009;28(1-2):15-33.

30. Yarden Y, Sliwkowski MX. Untangling the ErbB signalling network. Nat Rev Mol Cell Biol. 2001;2(2):127-137.

31. Feldner JC, Brandt BH. Cancer cell motility--on the road from c-erbB-2 receptor steered signaling to actin reorganization. Exp Cell Res. 2002;272(2):93-108.

32. Hirsch FR, Varella-Garcia M, Cappuzzo F. Predic tive value of EGFR and HER2 overexpression in advanced non-small-cell lung cancer. Oncogene. 2009;28(suppl 1):S32-S37.

33. Kopfstein L, Christofori G. Metastasis: cell autonomous mechanisms versus contributions by the tumor microenvironment. Cell Mol Life Sci. 2006;63(4):449-468.

34. Kotliarov Y, et al. High-resolution global genomic survey of 178 gliomas reveals novel regions of copy number alteration and allelic imbalances. Cancer Res. 2006;66(19):9428-9436.

35. Sanchez-Carbayo M, Socci ND, Lozano J, Saint F, Cordon-Cardo C. Defining molecular profiles of poor outcome in patients with invasive bladder cancer using oligonucleotide microarrays. J Clin Oncol. 2006;24(5):778-789.

36. Talantov D, et al. Novel genes associated with malignant melanoma but not benign melanocytic lesions. Clin Cancer Res. 2005;11(20):7234-7242.

37. Hoek KS, et al. Metastatic potential of melanomas defined by specific gene expression profiles with no BRAF signature. Pigment Cell Res. 2006;19(4):290-302.

38. Follenzi A, Naldini L. Generation of HIV-1 derived lentiviral vectors. Methods Enzymol. 2002;346:454-465.

39. Barberis $D$, et al. Plexin signaling hampers integrinbased adhesion, leading to Rho-kinase independent cell rounding, and inhibiting lamellipodia extension and cell motility. FASEB J. 2005;18(3):592-594. 